\title{
On the Performance of Coefficient of Variation Charts in the Presence of Measurement Errors
}

\author{
Kim Phuc Tran*1, Cédric Heuchenne ${ }^{2}$, and Narayanaswamy Balakrishnan ${ }^{3}$ \\ ${ }^{1}$ Ecole Nationale Supérieure des Arts et Industries Textiles, GEMTEX \\ Laboratory, BP 3032959056 Roubaix Cedex 1, France \\ ${ }^{2}$ HEC Management School, University of Liège, Liège 4000, Belgium \\ ${ }^{3}$ Department of Mathematics and Statistics, McMaster University, Hamilton, \\ Ontario L8S 4K1, Canada
}

September 4, 2018

\begin{abstract}
In the literature, coefficient of variation control charts have been introduced under the assumption of no measurement errors. However, measurement errors always exist in practice and they do affect the performance of control charts in the detection of an out of control situation. In this paper, we therefore study the performance of a coefficient of variation Shewhart type control chart (ShewhartCV chart) and also one-sided coefficient of variation EWMA type control charts (EWMA- $\gamma^{2}$ charts) using a model with linear covariates. Moreover, we propose and study the performance of a two-sided EWMA- $\gamma^{2}$ chart using a model with linear covariates. Several figures and tables are provided and analyzed to evaluate the statistical performance of these control charts for different sources of measurement errors. The obtained results show that the precision and accuracy errors significantly affect the performance of both the Shewhart-CV and EWMA- $\gamma^{2}$ control charts. An example illustrating the use of this study is finally presented.
\end{abstract}

Keywords: Measurement Errors; Coefficient of Variation; Textile manufacturing; Shewhart Control Chart; EWMA Control Charts; Markov chain.

\section{Introduction}

Statistical Process Control (SPC) is an important methodology in quality control. Control charts, the simplest type of on-line SPC procedure, are very useful tools for the detection and elimination of assignable causes shifting a process that is being monitored. In the SPC literature, Shewhart-type control chart for monitoring the coefficient

\footnotetext{
*kim-phuc.tran@ensait.fr (corresponding author)
} 
of variation $(\mathrm{CV})$, which is defined as the ratio of the population standard deviation to the population mean, has already been investigated by Kang et al. ${ }^{1}$; subsequently, several control charts have been developed in the literature for monitoring the coefficient of variation. For further details, see, for instance, Castagliola et al. ${ }^{2}$, Calzada and Scariano $^{3}$, Castagliola et al. ${ }^{4}$, Castagliola et al. ${ }^{5}$, Zhang et al. ${ }^{6}$, Castagliola et al. ${ }^{7}$, Castagliola et al. ${ }^{8}$, Amdouni et al. ${ }^{9}$, You et al. ${ }^{10}$ and Tran and Tran ${ }^{11}$, among others. For example, in textile manufacturing industry, the variation among tensile strength measurements from thin thread is significantly smaller than measurements taken from heavy thread due to the inherent physical properties of fiber. In this scenario, CV control charts are useful tools to monitor the process.

It is important to note that the control charts cited above for monitoring the CV are designed under the assumption that the measurements on the characteristic are made without error. However, in many industrial scenarios, there often exist significant measurement errors that affect the performance of control charts. Since Bennet ${ }^{12}$ investigated the effect of measurement errors on the Shewhart $\bar{X}$ chart, the consequences of the measurement errors on the performance of various control charts have been studied by a number of authors, including Kanazuka ${ }^{13}$, Linna and Woodall ${ }^{14}$, Linna et al. ${ }^{15}$, Maravelakis $^{16}$, Costa and Castagliola ${ }^{17}$, Maravelakis ${ }^{18}$, Hu et al. ${ }^{19}$, Noorossana and Zerehsaz ${ }^{20}$, Tran et al. ${ }^{21}$, Tran et al. ${ }^{22}$ and $\operatorname{Tran}^{23}$.

Quite recently, Yeong et al. ${ }^{24}$ investigated the effect of measurement errors on the one-sided CV charts. However, they have assumed that the ratios $\sigma_{M} / \sigma$ and $A / \mu$, which will be detailed in Section 3, are constants. This assumption may not be true in practice because when the process is out-of-control, these values do get changed. This study is designed to address the performance of the Shewhart-CV control chart proposed by Kang et al. ${ }^{1}$ and the one-sided EWMA- $\gamma^{2}$ control charts proposed by Castagliola et al. ${ }^{2}$ in the presence of measurement errors by assuming the same measurement errors model as the one in Linna and Woodall ${ }^{14}$. In addition, we propose and study the performance of two-sided EWMA- $\gamma^{2}$ chart in the presence of measurement errors. We also take into account the changes of these ratios when investigating the performance of Shewhart-CV and one-sided EWMA- $\gamma^{2}$ control charts. The organization of the rest of this article is as follows: In Section 2, the coefficient of variation distribution is introduced. In Section 3, the linear covariate error model for the coefficient of variation is introduced. Section 4 provides the formulas for the control limits and the performance metrics of the ShewhartCV control chart. In Section 5, the effect of measurement errors on the performance of Shewhart-CV control chart is investigated. Section 6 provides the formulas of the control limits and the performance metrics of the one-sided and two-sided EWMA- $\gamma^{2}$ control charts. In Section 7, the effect of measurement errors on the performance of the onesided and the two-sided EWMA- $\gamma^{2}$ control charts are investigated. Section 8 presents an example to illustrate the methods developed here, and finally some concluding remarks and recommendations are made in Section 9. 


\section{A brief review of distribution of the sample coefficient of variation}

The goal of this section is to present a brief review of the distribution of the sample coefficient of variation (CV). The population $\mathrm{CV} \gamma$ is defined as the ratio of the standard deviation $\sigma=\sigma(X)$ to the mean $\mu=E(X)$; i.e.,

$$
\gamma=\frac{\sigma}{\mu}
$$

Suppose we have a random sample of size $n$ of normal i.i.d. $(\mu, \sigma)$ random variables $\left\{X_{1}, \ldots, X_{n}\right\}$. Let $\bar{X}$ and $S$ be the sample mean and the sample standard deviation of this sample, i.e.,

$$
\bar{X}=\frac{1}{n} \sum_{i=1}^{n} X_{i}
$$

and

$$
S=\sqrt{\frac{1}{n-1} \sum_{i=1}^{n}\left(X_{i}-\bar{X}\right)^{2}} .
$$

Based on $\bar{X}$ and $S$, the sample coefficient of variation $\hat{\gamma}$ is defined as (Castagliola et al. ${ }^{2}$ )

$$
\hat{\gamma}=\frac{S}{\bar{X}}
$$

It is important to note that the probability distribution of the sample $\mathrm{CV} \hat{\gamma}$ has been studied in the literature by many authors; for further details, see McKay ${ }^{25}$, Hendricks and Robey ${ }^{26}$, Iglewicz et al. ${ }^{27}$, Iglewicz and Myers ${ }^{28}$, Warren ${ }^{29}$, Vangel ${ }^{30}$ and Reh and Scheffler ${ }^{31}$. Among these authors, Castagliola et al. ${ }^{2}$ showed that the c.d.f. (cumulative distribution function) $F_{\hat{\gamma}}(x \mid n, \gamma)$ of $\hat{\gamma}$ is

$$
F_{\hat{\gamma}}(x \mid n, \gamma)=1-F_{t}\left(\frac{\sqrt{n}}{x} \mid n-1, \frac{\sqrt{n}}{\gamma}\right)
$$

where $F_{t}\left(. \mid n-1, \frac{\sqrt{n}}{\gamma}\right)$ is the c.d.f. of the noncentral $t$ distribution with $n-1$ degrees of freedom and noncentrality parameter $\frac{\sqrt{n}}{\gamma}$. With some manipulations, inverting $F_{\hat{\gamma}}(x \mid n, \gamma)$ gives the inverse c.d.f. $F_{\hat{\gamma}}^{-1}(\alpha \mid n, \gamma)$ of $\hat{\gamma}$ as (Castagliola et al. ${ }^{2}$ )

$$
F_{\hat{\gamma}}^{-1}(\alpha \mid n, \gamma)=\frac{\sqrt{n}}{F_{t}^{-1}\left(1-\alpha \mid n-1, \frac{\sqrt{n}}{\gamma}\right)},
$$

where $F_{t}^{-1}\left(. \mid n-1, \frac{\sqrt{n}}{\gamma}\right)$ is the inverse c.d.f. of the noncentral $t$ distribution. 
Moreover, Castagliola et al. ${ }^{2}$ showed that $\frac{n}{\hat{\gamma}^{2}}$ follows a noncentral $F$ distribution with $(1, n-1)$ degrees of freedom and noncentrality parameter $\frac{n}{\gamma^{2}}$. Then, they deduced the c.d.f. $F_{\hat{\gamma}^{2}}(x \mid n, \gamma)$ of $\hat{\gamma}^{2}$ to be

$$
F_{\hat{\gamma}^{2}}(x \mid n, \gamma)=1-F_{F}\left(\frac{n}{x} \mid 1, n-1, \frac{n}{\gamma^{2}}\right),
$$

where $F_{F}\left(. \mid 1, n-1, \frac{n}{\gamma^{2}}\right)$ is the c.d.f. of the noncentral $F$ distribution. Castagliola et al. ${ }^{2}$ showed that the inverse c.d.f. $F_{\hat{\gamma}^{2}}^{-1}(\alpha \mid n, \gamma)$ of $\hat{\gamma}^{2}$ can be obtained as

$$
F_{\hat{\gamma}^{2}}^{-1}(\alpha \mid n, \gamma)=\frac{n}{F_{F}^{-1}\left(1-\alpha \mid 1, n-1, \frac{n}{\gamma^{2}}\right)},
$$

where $F_{F}^{-1}\left(. \mid 1, n-1, \frac{n}{\gamma^{2}}\right)$ is the inverse c.d.f. of the noncentral $F$ distribution.

\section{Linear covariate error model for the coefficient of varia- tion}

In this section, the linear covariate error model for the sample CV is introduced. Let us assume that, at times $i=1,2, \ldots$, the quality characteristic $X$ of $n>1$ consecutive items is equal to $\left\{X_{i, 1}, X_{i, 2}, \ldots, X_{i, n}\right\}$. We assume that $X_{i, j}$ 's are independent normal $\left(\mu_{0}+a \sigma_{0}, b \sigma_{0}\right)$ random variables, where $\mu_{0}$ and $\sigma_{0}$ are the nominal mean and standard deviation, respectively, both assumed known, while $a$ and $b$ are the standardized mean and standardized deviation shifts. The process has shifted if the process mean $\mu_{0}$ and/or the process standard deviation $\sigma_{0}$ have changed $(a \neq 0$ and/or $b \neq 1)$. As suggested by Linna and Woodall ${ }^{14}$, let us assume that the true quality characteristic $X_{i, j}$ with mean $\mu_{0}$ and variance $\sigma_{0}^{2}$ (when the process is in statistical control) is not directly observable, but can only be assessed from the results $\left\{X_{i, j, 1}^{*}, X_{i, j, 2}^{*}, \ldots, X_{i, j, m}^{*}\right\}$ of a set of $m \geq 1$ measurement operations with each $X_{i, j, k}^{*}$ being equal to (linear covariate error model)

$$
X_{i, j, k}^{*}=A+B X_{i, j}+\varepsilon_{i, j, k},
$$

where $A$ and $B$ are two known constants and $\varepsilon_{i, j, k}$ is a normal $\left(0, \sigma_{M}\right)$ random error term due to the measurement inaccuracy, which is independent of $X_{i, j}$. The smaller $\sigma_{M}$ is, the higher the precision is. The constants $A$ and $B$ for linear covariate error model can be estimated by using nonparametric estimation; see Li and Vuong ${ }^{32}$.

For subgroup $i=1,2, \ldots$, as $j=1,2, \ldots, n$ and $k=1,2, \ldots, m$, we have $m \times n$ observations $X_{i, j, k}^{*}$ and the mean $\bar{X}_{i, j}^{*}$ of the observable quantities $\left\{X_{i, j, 1}^{*}, X_{i, j, 2}^{*}, \ldots, X_{i, j, m}^{*}\right\}$ 
is equal to

$$
\begin{aligned}
\bar{X}_{i, j}^{*} & =\frac{1}{m} \sum_{k=1}^{m} X_{i, j, k}^{*} \\
& =\frac{1}{m} \sum_{k=1}^{m}\left(A+B X_{i, j}+\varepsilon_{i, j, k}\right) \\
& =A+B X_{i, j}+\frac{1}{m} \sum_{k=1}^{m} \varepsilon_{i, j, k} .
\end{aligned}
$$

It can then be easily shown that the mean $\mu^{*}=E\left(\bar{X}_{i, j}^{*}\right)$ and the standard deviation $\sigma^{*}=\sigma\left(\bar{X}_{i, j}^{*}\right)$ of $\bar{X}_{i, j}^{*}$ are equal to

$$
\begin{aligned}
\mu^{*} & =A+B\left(\mu_{0}+a \sigma_{0}\right), \\
\sigma^{*} & =\sqrt{B^{2} b^{2} \sigma_{0}^{2}+\frac{\sigma_{M}^{2}}{m}} .
\end{aligned}
$$

The coefficient of variation of the measured quantity $\bar{X}_{i, j}^{*}$ is then

$$
\gamma^{*}=\frac{\sigma^{*}}{\mu^{*}}=\frac{\sqrt{B^{2} b^{2} \sigma_{0}^{2}+\frac{\sigma_{M}^{2}}{m}}}{A+B\left(\mu_{0}+a \sigma_{0}\right)} .
$$

Let $\eta=\frac{\sigma_{M}}{\sigma_{0}}$ be the square root of the ratio of the measurement system variability. If we set $\gamma_{0}=\frac{\sigma_{0}}{\mu_{0}}$ and $\theta=\frac{A}{\mu_{0}}$, the coefficient of variation of the measured quantity $\bar{X}_{i, j}^{*}$ in (8) can be rewritten as

$$
\gamma^{*}=\frac{\sigma^{*}}{\mu^{*}}=\frac{\sqrt{B^{2} b^{2}+\frac{\eta^{2}}{m}}}{\theta+B\left(1+a \gamma_{0}\right)} \times \gamma_{0} .
$$

Let $\overline{\bar{X}}_{i}^{*}$ and $S_{i}^{*}$ be the sample mean and the sample standard deviation of $\bar{X}_{1, j}^{*}, \ldots, \bar{X}_{n, j}^{*}$, i.e.,

$$
\overline{\bar{X}}_{i}^{*}=\frac{1}{n} \sum_{j=1}^{n} \bar{X}_{i, j}^{*}
$$

and

$$
S_{i}^{*}=\sqrt{\frac{1}{n-1} \sum_{j=1}^{n}\left(\bar{X}_{i, j}^{*}-\overline{\bar{X}}_{j}^{*}\right)^{2}} .
$$

Then, the sample coefficient of variation $\hat{\gamma}_{i}^{*}$ is defined as

$$
\hat{\gamma}_{i}^{*}=\frac{S_{i}^{*}}{\overline{\bar{X}}_{i}^{*}} .
$$

The c.d.f. and i.d.f. of $\hat{\gamma}^{*}$ can be obtained from (1) and (2) by simply replacing $\gamma$ by $\gamma^{*}$ as defined in (9), i.e., the c.d.f. $F_{\gamma^{*}}\left(x \mid n, \gamma^{*}\right)$ and i.d.f. $F_{\gamma^{*}}^{-1}\left(\alpha \mid n, \gamma^{*}\right)$ of $\hat{\gamma}^{*}$ are given by 


$$
F_{\gamma^{*}}\left(x \mid n, \gamma^{*}\right)=1-F_{t}\left(\frac{\sqrt{n}}{x} \mid n-1, \frac{\sqrt{n}}{\gamma^{*}}\right)
$$

and

$$
F_{\gamma^{*}}^{-1}\left(\alpha \mid n, \gamma^{*}\right)=\frac{\sqrt{n}}{F_{t}^{-1}\left(1-\alpha \mid n-1, \frac{\sqrt{n}}{\gamma^{*}}\right)}
$$

Similarly, the c.d.f. and i.d.f. of $\hat{\gamma}^{* 2}$ can be obtained from (3) and (4) by simply replacing $\gamma$ by $\gamma^{*}$ as defined in (9), i.e., the c.d.f. $F_{\gamma^{* 2}}\left(x \mid n, \gamma^{*}\right)$ and i.d.f. $F_{\gamma^{* 2}}^{-1}\left(\alpha \mid n, \gamma^{*}\right)$ of $\hat{\gamma}^{* 2}$ are given by

$$
F_{\gamma^{* 2}}\left(x \mid n, \gamma^{*}\right)=1-F_{F}\left(\frac{n}{x} \mid 1, n-1, \frac{n}{\gamma^{* 2}}\right)
$$

and

$$
F_{\gamma^{* 2}}^{-1}\left(\alpha \mid n, \gamma^{*}\right)=\frac{n}{F_{F}^{-1}\left(1-\alpha \mid 1, n-1, \frac{n}{\gamma^{* 2}}\right)} .
$$

\section{Implementation of the Shewhart-CV control chart with measurement errors}

The control limits of Shewhart-CV control chart in the presence of measurement errors are defined as

$$
\begin{aligned}
L C L & =F_{\gamma^{*}}^{-1}\left(\frac{\alpha_{0}}{2} \mid n, \gamma_{0}^{*}\right), \\
U C L & =F_{\gamma^{*}}^{-1}\left(1-\frac{\alpha_{0}}{2} \mid n, \gamma_{0}^{*}\right),
\end{aligned}
$$

where $F_{\gamma^{*}}^{-1}($.$) is the inverse c.d.f. of \hat{\gamma}^{*}$ defined in (11) and $\alpha$ is the desired false alarm probability for the control chart. In (14) and (15), the value of $\gamma_{0}^{*}$ is computed as

$$
\gamma_{0}^{*}=\frac{\sigma^{*}}{\mu^{*}}=\frac{\sqrt{B^{2}+\frac{\eta^{2}}{m}}}{B+\theta} \times \gamma_{0},
$$

where $\gamma_{0}$ is the in-control CV.

The central line $C L$ of the Shewhart-CV chart can be defined as the median value, i.e.,

$$
C L=F_{\gamma^{*}}^{-1}\left(0.5 \mid n, \gamma_{0}^{*}\right) .
$$

Now, let us assume that the occurrence of an out-of-control condition shifts the incontrol CV $\gamma_{0}$ to $\gamma_{1}=\tau \times \gamma_{0}$, where $\tau>0$ is the size of the shift. Then, it can be easily shown that

$$
\gamma_{1}=\frac{b}{1+a \gamma_{0}}=\tau \times \gamma_{0}
$$


or

$$
\frac{b}{\tau}=1+a \gamma_{0}
$$

From (18) and (9), the out-of-control coefficient of variation of the measured quantity $\bar{X}_{i, j}^{*}$ can be rewritten as

$$
\gamma_{1}^{*}=\frac{\sigma^{*}}{\mu^{*}}=\frac{\sqrt{B^{2} b^{2}+\frac{\eta^{2}}{m}}}{\theta+\frac{B b}{\tau}} \times \gamma_{0} .
$$

As with any other Shewhart control chart, the run length of the Shewhart-CV control chart with measurement errors is defined as

$$
\beta=F_{\gamma^{*}}\left(U C L \mid n, \gamma_{1}^{*}\right)-F_{\hat{\gamma}}\left(L C L \mid n, \gamma_{1}^{*}\right) .
$$

It is straightforward to get an expression for the out-of-control $A R L$ as

$$
A R L=\frac{1}{1-\beta} .
$$

\section{The effect of measurement errors on the Shewhart-CV control chart}

In this Section, we investigate the performance of the Shewhart-CV control chart in the presence of measurement errors. When the process is in-control, the $A R L$ is denoted by $A R L_{0}$ and here we set $\alpha=0.0027$ corresponding to $A R L_{0}=370.4$. Without loss of generality, we assume in the remaining part of this section that $b=1$. From Section 4 , for fixed values of $m, n, B$ and $\eta$, we can obtain the $A R L$ and $S D R L$ values of the Shewhart-CV chart with linear covariate error model.

Table 1 shows the values of the probability control limits $(L C L, U C L)$ for the values of $n \in\{5,7,10,15\}, \eta \in\{0.1,0.28\}, \theta \in\{0.01,0.05\}, \gamma_{0} \in\{0.05,0.1,0.15,0.2\}, B=1$, $m=1$ and $A R L_{0}=370.4$. The specific value of $\eta=0.28$ is motivated by assuming an acceptable value for the signal-to-noise ratio

$$
S N R=\sqrt{\frac{\frac{2}{1+\eta^{2}}}{1-\frac{1}{1+\eta^{2}}}}=\frac{\sqrt{2}}{\eta},
$$

which is a measure of performance of the precision adequacy of the measurement system; see Montgomery ${ }^{33}$. As discussed in Tran et al. ${ }^{21}$, assuming $\eta=0.28$ corresponds to $S N R=5$, which is the lower bound value to get an acceptable precision of the measurement system.

\section{INSERT TABLE 1 ABOUT HERE}


Some simple conclusions can be drawn from Table 1 as follows:

- In general, given $n, B$ and $\gamma_{0}$, the values of $L C L$ and $U C L$ depend on $(\eta, \theta)$. In particular, for smaller values of $(\eta, \theta)$, the values of $L C L$ and $U C L$ are smaller. For example, when $n=5, B=1$ and $\gamma_{0}=0.05$, we have $L C L=0.0081$ and $U C L=0.1053$ when $(\eta, \theta)=(0.10,0.01)$, and $L C L=0.0080$ and $U C L=0.1047$ when $(\eta, \theta)=(0.28,0.05)$;

- In general, given $n, \gamma_{0}, \eta$ and $\theta$, the values of $L C L$ and $U C L$ depend on $B$. In particular, for smaller $B$, the values of $L C L$ and $U C L$ are smaller. For example, when $n=5, \gamma_{0}=0.1$ and $(\eta, \theta)=(0.10,0.01)$, we have $L C L=0.0161$ and $U C L=0.2130$ when $B=1$, and $L C L=0.0162$ and $U C L=0.2137$ when $B=5$.

Table 2 shows the out-of-control $A R L_{1}$ values for the Shewhart-CV for different combinations of the precision error ratio $\eta \in\{0,0.1,0.2,0.3,0.5,1.0\}, \gamma_{0} \in\{0.05,0.1,0.2\}$, $\tau \in\{0.5,0.65,0.8,1.25,1.5,2\}$ and $n \in\{5,7,10,15\}$ when $m=1, \theta=0.05$ and $B=1$.

\section{INSERT TABLE 2 ABOUT HERE}

The obtained results show that, for fixed values of $n, \eta, \gamma_{0}, m=1$ and $B=1$, the smaller the precision error ratio $\eta$ is, the faster the control chart is in detecting the out-of-control condition, demonstrating the negative effect of measurement errors on the performance of Shewhart-CV chart. However, when $\eta \leq 0.3$, the values of $A R L$ in the presence of a precision error are not significantly larger than the value of $A R L$ without the measurement error. For instance, when $n=5, B=1, m=1, \gamma_{0}=0.05$ and $\tau=0.7$, we have $A R L=148.13$ for $\eta=0$ and $A R L=148.15$ for $\eta=0.3$ (see Table 2). So, we can conclude that the precision error does not significantly affect the performance of the Shewhart-CV control chart with a normal measurement system.

Table 3 shows the out-of-control $A R L_{1}$ values for the Shewhart-CV for different combinations of the precision error ratio $\theta \in\{0,0.01,0.02,0.03,0.04,0.05\}, \gamma_{0} \in\{0.05,0.1,0.2\}$, $\tau \in\{0.5,0.65,0.8,1.25,1.5,2\}$ and $n \in\{5,7,10,15\}$ when $m=1, \eta=0.28$ and $B=1$.

\section{INSERT TABLE 3 ABOUT HERE}

The obtained results show that, for fixed values of $n, \tau, \gamma_{0}, m=1$ and $B=1$, the performance of the Shewhart-CV control chart is influenced by the accuracy error, measured by $\theta$. In most cases, the larger the values of $\theta$, the faster the control charts are in detecting the out-of-control condition. For instance, when $n=7, \gamma_{0}=0.1, B=1$, $m=1$ and $\tau=0.7$, we have $A R L=69.93$ for $\theta=0$, and $A R L=76.32$ for $\theta=0.05$ (see Table 3 ). We can thus conclude that the accuracy error significantly affects the performance of the Shewhart-CV control chart for the usual levels of accuracy errors provided by calibrated gauges. 


\section{INSERT TABLE 4 ABOUT HERE}

Table 4 shows the performances of Shewhart-CV chart under linear covariate error model for different combinations of $B \in\{1,2,3,4,5\}, \tau \in\{0.5,0.65,0.8,1.25,1.5,2\}$, $\gamma_{0} \in\{0.05,0.1,0.2\}$ and $n \in\{5,7,10,15\}$ when $m=1$ and $\eta=0.28, \theta=0.05$. It can be noted from Table 4 that, for fixed values of $n, \tau, \eta, \theta$ and $m$, the value of $B$ significantly affects the performance of the Shewhart-CV control chart. For instance, when $n=5$, $\eta=0.28, \theta=0.05, \gamma_{0}=0.1, m=1$ and $\tau=0.7$, we have $A R L=148.9$ for $B=1$, and $A R L=141.5$ for $B=5$ (see Table 4 ).

As discussed in Linna and Woodall ${ }^{14}$, it is better to take multiple measurements per item in each sample to compensate for the effect of measurement errors. The performance of the Shewhart-CV chart under linear covariate error model is shown in Table 5 for different combinations of $m \in\{1,3,5,7,10\}, \tau \in\{0.5,0.65,0.8,1.25,1.5,2\}$, $\gamma_{0} \in\{0.05,0.1,0.2\}$ and $n \in\{3,5,7,9\}$ when $B=1$ and $\eta=0.28$. For fixed values of $n, \tau, B$ and $\eta$, as the number $m$ of measurements per item increases, the value of $A R L$ decreases, demonstrating the positive effect of the number of repeated measurements $m$ per item on the performance of the Shewhart-CV chart. For instance, when $n=10$, $\eta=0.28$ and $\tau=0.7, \gamma_{0}=0.1$, we have $A R L=34.14$ for $m=1$, and $A R L=31.21$ for $m=5$ (see Table 5 ).

\section{INSERT TABLE 5 ABOUT HERE}

\section{Implementation of the EWMA- $\gamma^{2}$ control charts with measurement errors}

In this Section, we propose a two-sided EWMA control chart for monitoring $\hat{\gamma}^{2}$ (denoted as two-sided EWMA- $\gamma^{2}$ ) with measurement errors. We also study the performance of one-sided EWMA- $\gamma^{2}$ control charts proposed by Castagliola et al. ${ }^{2}$ in the presence of measurement errors. The two-sided EWMA- $\gamma^{2}$ control chart is useful when the direction of the shift is not known in advance or when the detection of upward and downward shifts is of equal importance. It is important to note that there are no closed-form expressions for $\mu_{0}\left(\hat{\gamma}^{* 2}\right)$ and $\sigma_{0}\left(\hat{\gamma}^{* 2}\right)$; see Castagliola et al. ${ }^{2}$ for more details. In this case, Breunig ${ }^{34}$ provided accurate approximations as

$$
\begin{aligned}
\mu_{0}\left(\hat{\gamma}^{* 2}\right) & =\gamma_{0}^{* 2}\left(1-\frac{3 \gamma_{0}^{* 2}}{n}\right) \\
\sigma_{0}\left(\hat{\gamma}^{* 2}\right) & =\sqrt{\gamma_{0}^{* 4}\left(\frac{2}{n-1}+\gamma_{0}^{* 2}\left(\frac{4}{n}+\frac{20}{n(n-1)}+\frac{75 \gamma_{0}^{* 2}}{n^{2}}\right)\right)-\left(\mu_{0}\left(\hat{\gamma}^{* 2}\right)-\gamma_{0}^{* 2}\right)^{2}},
\end{aligned}
$$

where $\gamma_{0}^{*}$ is as defined in (9). 
The two-sided EWMA- $\gamma^{2}$ is defined as

$$
S_{i}^{*}=(1-\lambda) S_{i-1}^{*}+\lambda \hat{\gamma}_{i}^{* 2},
$$

with $S_{0}^{*}=\mu_{0}\left(\hat{\gamma}^{* 2}\right)$ as an initial value. The control limits of a two-sided EWMA control chart in the presence of measurement errors are defined as

$$
\begin{aligned}
L C L_{\mathrm{EWMA}-\gamma^{2}} & =\mu_{0}\left(\hat{\gamma}^{* 2}\right)-K \sqrt{\frac{\lambda}{2-\lambda}} \sigma_{0}\left(\hat{\gamma}^{* 2}\right), \\
U C L_{\mathrm{EWMA}-\gamma^{2}} & =\mu_{0}\left(\hat{\gamma}^{* 2}\right)+K \sqrt{\frac{\lambda}{2-\lambda}} \sigma_{0}\left(\hat{\gamma}^{* 2}\right),
\end{aligned}
$$

where $K>0$ comes from solving $A R L\left(\gamma_{0}^{*}, \lambda, n, m, B, \eta, K\right)=A R L_{0}$ and $\lambda$ is the smoothing constant of the two-sided EWMA- $\gamma^{2}$ chart.

In order to detect shifts on a specific direction, one-sided schemes are more effective than two-sided ones (see also Castagliola et al. ${ }^{2}$ ). According to Castagliola et al. ${ }^{2}$, the following two separate one-sided EWMA charts in the presence of measurement errors may be used:

- an upward EWMA chart (denoted as "upward EWMA- $\gamma^{2}$ ") is given by

$$
Z_{i}^{*+}=\max \left(\mu_{0}\left(\hat{\gamma}^{* 2}\right),\left(1-\lambda^{+}\right) Z_{i-1}^{*+}+\lambda^{+} \hat{\gamma}_{i}^{* 2}\right),
$$

with $Z_{0}^{*+}=\mu_{0}\left(\hat{\gamma}^{* 2}\right)$ as an initial value and with the corresponding upper control limit

$$
U C L_{\mathrm{EWMA}-\gamma^{2}}=\mu_{0}\left(\hat{\gamma}^{* 2}\right)+K^{+} \sqrt{\frac{\lambda^{+}}{2-\lambda^{+}}} \sigma_{0}\left(\hat{\gamma}^{* 2}\right) ;
$$

- a downward EWMA chart (denoted as "downward EWMA- $\gamma^{2}$ ") is given by

$$
Z_{i}^{*-}=\min \left(\mu_{0}\left(\hat{\gamma}^{* 2}\right),\left(1-\lambda^{-}\right) Z_{i-1}^{*-}+\lambda^{-} \hat{\gamma}_{i}^{* 2}\right),
$$

with $Z_{0}^{*-}=\mu_{0}\left(\hat{\gamma}^{* 2}\right)$ as an initial value and with the corresponding lower control limit

$$
L C L_{\mathrm{EWMA}-\gamma^{2}}=\mu_{0}\left(\hat{\gamma}^{* 2}\right)-K^{-} \sqrt{\frac{\lambda^{-}}{2-\lambda^{-}}} \sigma_{0}\left(\hat{\gamma}^{* 2}\right),
$$

where $\mu_{0}\left(\hat{\gamma}^{* 2}\right)$ and $\sigma_{0}\left(\hat{\gamma}^{* 2}\right)$ are the mean and standard deviation of $\hat{\gamma}^{2}$ when the process is in-control, and $\lambda^{+}\left(\lambda^{-}\right)$and $K^{+}\left(K^{-}\right)$are the smoothing constant and chart coefficient of the upward (downward) EWMA- $\gamma^{2}$ chart.

Once the control chart parameters (in our case, $\lambda^{-}$or $\lambda^{+}$and $K^{-}$or $K^{+}$for the one-sided EWMA- $\gamma^{2}$ control charts; and $\lambda$ and $K$ for the two-sided EWMA- $\gamma^{2}$ control chart), are defined, the $A R L$ can be numerically evaluated for particular shifts $a$ and $b$, 
from an in-control value $\gamma_{0}^{*}$ to an out-of-control value $\gamma_{1}^{*}$. Here, to calculate the $A R L$ of one-sided and two-sided EWMA- $\gamma^{2}$ control charts, we use a Markov-chain approximation based on a flexible and relatively easy to use procedure given in Castagliola et al. ${ }^{2}$, originally proposed by Brook and Evans ${ }^{35}$; see Castagliola et al. ${ }^{2}$ for more details.

As in Castagliola et al. $^{2}$, the design procedure of one-sided EWMA- $\gamma^{2}$ charts is implemented by determining the optimal combinations $\left(\lambda^{+*}, K^{+*}\right)$ or $\left(\lambda^{-*}, K^{-*}\right)$ for particular shifts $a$ and $b$, such that

- for upward EWMA- $\gamma^{2}$ chart

$$
\left(\lambda^{+*}, K^{+*}\right)=\underset{\left(\lambda^{+}, K^{+}\right)}{\operatorname{argmin}} A R L\left(\gamma_{0}^{*}, \gamma_{1}^{*}, \lambda^{+}, K^{+}, n, m, B, \eta\right),
$$

subject to the constraint

$$
A R L\left(\gamma_{0}^{*}, \lambda^{*}, K^{+*}, n, m, B, \eta\right)=A R L_{0},
$$

- for downward EWMA- $\gamma^{2}$ chart

$$
\left(\lambda^{-*}, K^{-*}\right)=\underset{\left(\lambda^{-}, K^{-}\right)}{\operatorname{argmin}} A R L\left(\gamma_{0}^{*}, \gamma_{1}^{*}, \lambda^{-}, K^{-}, n, m, B, \eta\right),
$$

subject to the constraint

$$
A R L\left(\gamma_{0}^{*}, \lambda^{-*}, K^{-*}, n, m, B, \eta\right)=A R L_{0} .
$$

Similarly, the design procedure of two-sided EWMA- $\gamma^{2}$ chart is implemented by determining the optimal combination $\left(\lambda^{*}, K^{*}\right)$ for particular shifts $a$ and $b$, such that

$$
\left(\lambda^{*}, K^{*}\right)=\underset{(\lambda, K)}{\operatorname{argmin}} A R L\left(\gamma_{0}^{*}, \gamma_{1}^{*}, \lambda, K, n, m, B, \eta\right),
$$

subject to the constraint

$$
A R L\left(\gamma_{0}^{*}, \lambda^{*}, K^{*}, n, m, B, \eta\right)=A R L_{0} .
$$

In this study, we use a non-linear equation solver along with an optimization achieved through an algorithm developed in Scicoslab software; see Tran and Tran ${ }^{11}$ for more details.

\section{The effect of measurement errors on the EWMA- $\gamma^{2}$ con- trol charts}

In this Section, we investigate the performance of the proposed two-sided EWMA- $\gamma^{2}$ and the EWMA- $\gamma^{2}$ control charts proposed by Castagliola et al. ${ }^{2}$ in the presence of measurement errors. When the process is in-control, we set $A R L_{0}=370.4$. Without 
loss of generality, we assume in the remaining part of this section that $b=1$. From Section 4, for fixed values of $m, n, B, \lambda, K, a$ and $\eta$, we can obtain the $A R L$ and $S D R L$ values of the proposed two-sided EWMA- $\gamma^{2}$ and the one-sided EWMA- $\gamma^{2}$ control charts.

Table 6 shows optimal values $\lambda^{*}$ and $K^{*}$ of two-sided EWMA- $\gamma^{2}$ control charts in the presence of measurement errors for the values of $n \in\{5,7,10,15\}, \eta \in\{0.1,0.28\}$, $\theta=0.0 .05, \gamma_{0} \in\{0.05,0.1,0.2\}, B=1, m=1$ and $A R L_{0}=370.4$. For the sake of brevity, similar tables showing optimal values $\lambda^{*}$ and $K^{*}$ of two-sided EWMA- $\gamma^{2}$ control charts for other scenarios are not presented here, but are available upon request from the authors. For example, when $n=5, B=1, \gamma_{0}=0.05, \tau=0.8$ and $\eta=0.2$, we have $\lambda^{*}=0.0302, K^{*}=2.2973$ when $\gamma_{0}=0.05$, and $\lambda^{*}=0.0274, K^{*}=2.2738$ when $\gamma_{0}=0.1$. In this case, Table 7 shows the out-of-control $A R L_{1}$ values for the two-sided EWMA- $\gamma^{2}$.

\section{INSERT TABLE 6 ABOUT HERE}

\section{INSERT TABLE 7 ABOUT HERE}

The obtained results show that the performance of two-sided EWMA- $\gamma^{2}$ control chart is influenced by the values of $\eta$ with a similar behaviour as for the Shewhart-CV control chart discussed earlier. In most cases, the two-sided EWMA- $\gamma^{2}$ control chart outperforms the Shewhart-CV chart. For example, when $n=5, B=1, \gamma_{0}=0.05$, $\tau=0.8$ and $\theta=0.01, \gamma_{0}=0.05$ and $\eta=0.2$, we have $A R L_{1}=319.60$ for Shewhart$\mathrm{CV}$ chart and $A R L_{1}=28.08$ for two-sided EWMA- $\gamma^{2}$ control chart (see Tables 2 and 7 ).

Table 3 shows the out-of-control $A R L_{1}$ values for the two-sided EWMA- $\gamma^{2}$ for different combinations of the precision error ratio $\theta \in\{0,0.01,0.02,0.03,0.04,0.05\}$, $\gamma_{0} \in\{0.05,0.1,0.2\}, \tau \in\{0.5,0.65,0.8,1.25,1.5,2\}$ and $n \in\{5,7,10,15\}$ when $m=1$, $\eta=0.28$ and $B=1$.

\section{INSERT TABLE 8 ABOUT HERE}

The obtained results show that the performance of the two-sided EWMA- $\gamma^{2}$ control chart is influenced by the values of $\theta$ with a similar behaviour as for the Shewhart-CV control chart discussed earlier. In most cases, the two-sided EWMA- $\gamma^{2}$ control chart outperforms the Shewhart-CV chart. For example, when $n=5, B=1, \gamma_{0}=0.05$, $\tau=0.8, \eta=0.28, \gamma_{0}=0.05$ and $\theta=0.02$, we have $A R L_{1}=312.97$ for Shewhart-CV chart and $A R L_{1}=27.32$ for two-sided EWMA- $\gamma^{2}$ control chart (see Tables 3 and 8).

\section{INSERT TABLE 9 ABOUT HERE}

Table 9 shows the performances of the two-sided EWMA- $\gamma^{2}$ chart under linear covariate error model for different combinations of $B \in\{1,2,3,4,5\}, \tau \in\{0.5,0.65,0.8,1.25,1.5,2\}$, 
$\gamma_{0} \in\{0.05,0.1,0.2\}$ and $n \in\{5,7,10,15\}$ when $m=1$ and $\eta=0.28, \theta=0.05$. It can be noted from Table 9 that the performance of the two-sided EWMA- $\gamma^{2}$ control chart is influenced by the values of $B$ with a similar behaviour as for the Shewhart-CV control chart discussed earlier. Moreover, in most cases, the two-sided EWMA- $\gamma^{2}$ control chart outperforms the Shewhart-CV chart. For example, when $n=5, \gamma_{0}=0.05, \tau=0.8$, $\eta=0.28, \gamma_{0}=0.05, \theta=0.01, B=2$, we have $A R L_{1}=314.07$ for Shewhart-CV chart and $A R L_{1}=27.43$ for two-sided EWMA- $\gamma^{2}$ control chart (see Tables 4 and 9).

The performance of the two-sided EWMA- $\gamma^{2}$ chart under linear covariate error model is shown in Table 10 for different combinations of $m \in\{1,3,5,7,10\}, \tau \in$ $\{0.5,0.65,0.8,1.25,1.5,2\}, \gamma_{0} \in\{0.05,0.1,0.2\}$ and $n \in\{3,5,7,9\}$ when $B=1$ and $\eta=0.28$. For fixed values of $n, \tau, B$ and $\eta$, as the number $m$ of measurements per item increases, the value of $A R L$ decreases, demonstrating the positive effect of the number of repeated measurements $m$ per item on the performance of the two-sided EWMA- $\gamma^{2}$ chart with similar behaviour as for the Shewhart-CV control chart discussed earlier. Moreover, in most cases, the two-sided EWMA- $\gamma^{2}$ control chart outperforms the Shewhart-CV chart. For instance, when $n=5, \eta=0.28, \theta=0.01, \tau=0.8, m=5$ and $\gamma_{0}=0.05$, we have $A R L_{1}=319.59$ for Shewhart-CV chart and $A R L_{1}=27.43$ for two-sided EWMA- $\gamma^{2}$ control chart (see Tables 5 and 10).

\section{INSERT TABLE 10 ABOUT HERE}

Similar tables showing the effect of measurement errors for the one-sided EWMA- $\gamma^{2}$ charts are not presented here, but are available upon request from the authors. The performance of one-sided EWMA- $\gamma^{2}$ control charts is influenced by measurement errors with a similar behaviour as for the Shewhart-CV and two-sided EWMA- $\gamma^{2}$ control charts discussed earlier. But, in general, quality practitioners often have an interest in detecting a range of shifts $\Omega=[a, b]$, but with no preference for any particular size of the process shift (for instance, see Chen and Chen ${ }^{36}$ and Celano et al. ${ }^{37}$ ). In this case, the statistical performance of the corresponding chart can be evaluated through the EARL (Expected Average Run Length) defined as

$$
E A R L=\int_{\Omega} A R L \times f_{\tau}(\tau) \mathrm{d} \tau
$$

with $f_{\tau}(\tau)=\frac{1}{b-a}$ for $\tau \in \Omega=[a, b]$ and $A R L$ is as defined in (21). In the following section, we will consider two different ranges of shifts: $\Omega_{D}=[0.9,1)$ corresponding to a decreasing case for $\tau$ and $\Omega_{I}=[1,1.1)$ corresponding to an increasing case for $\tau$.

In this case, we find unique optimal couples $\left(\lambda^{-*}, K^{-*}\right)$ and $\left(\lambda^{+*}, K^{+*}\right)$ of the onesided EWMA- $\gamma^{2}$ charts such that

$$
\left(\lambda^{*}, K^{*}\right)=\underset{(\lambda, K)}{\operatorname{argmin}} \operatorname{EARL}\left(\gamma_{0}^{*}, \gamma_{1}^{*}, \lambda, K, n, m, B, \eta\right),
$$


subject to the constraint

$$
\operatorname{EARL}\left(\gamma_{0}^{*}, \lambda, K, n\right)=A R L\left(\gamma_{0}^{*}, \lambda, K, n, m, B, \eta\right)=A R L_{0},
$$

The optimal design parameters for the one-sided EWMA- $\gamma^{2}$ charts in the presence of measurement errors is presented in Table 11. For example, when $n=5, B=1$ and $\gamma_{0}=0.05$, we have $\lambda^{*}=0.0501$ and $K^{*}=2.1425$ for the downward EWMA- $\gamma^{2}$ when $(\eta, \theta)=(0.10,0.01)$.

\section{INSERT TABLE 11 ABOUT HERE}

Here, in order to evaluate the overall performance of the one-sided EWMA- $\gamma^{2}$ charts with linear covariate error model, we will use $E A R L$ values. In the following section, we will consider the specific range of shifts $\Omega=[0.5,1)$ (decreasing case, denoted by $\Omega_{D}$ ) and $\Omega=(1,2]$ (increasing case, denoted by $\Omega_{I}$ ).

The values of $E A R L$ of EWMA- $\gamma^{2}$ control charts are plotted in Figure 1 for $\eta \in[0,1]$, $\theta \in[0,0.05], \Omega_{D}=[0.5,1)$ and $\Omega_{I}=(1,2], n \in\{5,15\}, m=1$ and $B=1$.

\section{INSERT FIGURE 1 ABOUT HERE}

The obtained results show that, for fixed values of $n, \gamma_{0}, m=1$ and $B=1$, the smaller the precision error ratio $\eta$ and the accuracy error $\theta$ are, the faster the control chart is in detecting the out-of-control condition, demonstrating the negative effect of measurement errors on the performance of one-sided EWMA- $\gamma^{2}$ chart. However, when $\eta \leq 0.3$ and $\theta \leq 0.0115$, the values of $E A R L$ in the presence of a precision error are not significantly larger than the value of $E A R L$ without measurement error. For instance, when $n=5$, $B=1, m=1, \gamma_{0}=0.05$, we have $E A R L=36.3$ for $\eta=0, \theta=0$, and $A R L=36.7$ for $\eta=0.3, \theta=0.01$ for the downward EWMA- $\gamma^{2}$ (see Figure 1). So, we can conclude that the precision error does not significantly affect the performance of the one-sided EWMA- $\gamma^{2}$ control charts with a normal measurement system. But, the accuracy error significantly affects the performance of the one-sided EWMA- $\gamma^{2}$ control charts for the usual levels of accuracy errors provided by calibrated gauges. For instance, when $n=5$, $B=1, m=1, \gamma_{0}=0.05$, we have $E A R L=36.3$ for $\eta=0$ and $\theta=0$, and $A R L=38.2$ for $\eta=0$ and $\theta=0.05$ for the downward EWMA- $\gamma^{2}$ (see Figure 1).

The values of $E A R L$ of the one-sided EWMA- $\gamma^{2}$ control charts are plotted in Figure 2 for $\eta=0.28, \theta=0.05, \Omega_{D}=[0.5,1)$ and $\Omega_{I}=(1,2], n \in\{3,5,7,9\}, m=1$ and $B \in[1,5]$.

\section{INSERT FIGURE 2 ABOUT HERE}

It can be noted from Figure 2 that, for fixed values of $n, \tau, \eta, \theta$ and $m$, the value of $B$ significantly affects the performance of the one-sided EWMA- $\gamma^{2}$ control charts. For 
instance, when $n=5, \eta=0.28, \theta=0.05, \gamma_{0}=0.05$ and $m=1$, we have $E A R L=38.2$ for $B=1$, and $A R L=36.7$ for $B=5$ for the downward EWMA- $\gamma^{2}$ (see Figure 2).

The values of $E A R L$ of EWMA- $\gamma^{2}$ control charts are plotted in Figure 3 for $\eta=0.28$, $\theta=0.05, \Omega_{D}=[0.5,1)$ and $\Omega_{I}=(1,2], n \in\{3,5,7,9\}, B=1$ and $m \in[1,10]$.

\section{INSERT FIGURE 3 ABOUT HERE}

For fixed values of $n, B, \eta$ and $\theta$, as the number $m$ of measurements per item increases, the values of $E A R L$ of the one-sided EWMA- $\gamma^{2}$ control charts slightly decrease. For instance, when $n=5, \eta=0.28, \theta=0.05$ and $\gamma_{0}=0.05$, we have $A R L=38.2$ for $m=1$ and $A R L=38.2$ for $m=10$ for the downward EWMA- $\gamma^{2}$ (see Figure 3).

\section{Illustrative example}

In this Section, we discuss the implementation of Shewhart-CV chart in the presence of measurement errors. Let us consider a sintering process in an Italian company that manufactures sintered mechanical parts. The context of the example presented here is similar to the one introduced in Castagliola et al. ${ }^{2}$. As discussed in Castagliola et al. ${ }^{2}$, the process manufactures parts, to ensure a pressure test drop time $T_{p d}$ from 2 bar to 1.5 bar larger than $30 \mathrm{sec}$, are seen as a quality characteristic related to the pore shrinkage. They stated that the preliminary regression study relating $T_{p d}$ to the quantity $Q_{C}$ of molten copper has demonstrated the presence of a constant proportionality $\sigma_{p d}=\gamma_{p d} \times \mu_{p d}$ between the standard deviation of the pressure drop time and its mean. The quality practitioner decided to monitor the coefficient of variation $\gamma_{p d}=\sigma_{p d} / \mu_{p d}$ to detect changes in the process variability.

We assume that, from a Phase I dataset, the following quantity has been estimated $\hat{\gamma}_{0}=0.01$. Concerning the parameters of the linear covariate error model, we assume $\eta=0.28, \theta=0, B=1, m=1$. For phase II, the dataset with a sample size $n=5$ is simulated and presented in Table 12. The process is assumed to run in-control up to sample \#10. Then, between samples \#10 and \#11, we have simulated the occurrence of an assignable cause shifting $\gamma_{p d}$ from $\gamma_{p d}=0.01$ to $\gamma_{p d}=0.011$, i.e., $\tau=1.1$.

\section{INSERT TABLE 12 ABOUT HERE}

INSERT FIGURE 4 ABOUT HERE

INSERT FIGURE 5 ABOUT HERE

INSERT FIGURE 6 ABOUT HERE 
The values of $\hat{\gamma}_{i}^{*}, \hat{\gamma}_{i}^{* 2}, Z_{i}^{*}$ (for upward EWMA- $\gamma^{2}$ chart) and $S_{i}^{*}$ (for two-sided EWMA- $\gamma^{2}$ chart) are presented in Table 12. Based on (25), the control limits of Shewhart-CV control chart $\left(A R L_{0}=370.4\right)$ with measurement errors are equal to

$$
\begin{aligned}
L C L & =0.00169, \\
U C L & =0.02192 .
\end{aligned}
$$

The optimal parameters $\lambda^{*}$ and $K^{*}$ of upward EWMA- $\gamma^{2}$ chart are found by the optimizing algorithm to be $\lambda^{*}=0.05$ and $K^{*}=2.6743$. Based on (26), the control limits of upward EWMA- $\gamma^{2}$ chart $\left(A R L_{0}=370.4\right)$ with measurement errors are equal to

$$
U C L_{\mathrm{EWMA}-\gamma^{2}}=0.000140 .
$$

The optimal parameters $\lambda^{*}$ and $K^{*}$ of two-sided EWMA- $\gamma^{2}$ chart are found by the optimizing algorithm to be $\lambda^{*}=0.064038$ and $K^{*}=2.588766$. Based on (14) and (15), the control limits of two-sided EWMA- $\gamma^{2}$ chart $\left(A R L_{0}=370.4\right)$ with measurement errors are equal to

$$
\begin{aligned}
L C L_{\mathrm{EWMA}-\gamma^{2}} & =0.000072, \\
U C L_{\mathrm{EWMA}-\gamma^{2}} & =0.000144 .
\end{aligned}
$$

The values of $\hat{\gamma}_{i}^{*}$ and the control limits of Shewhart-CV chart are plotted in Figure 4. This figure confirms that from sample \#12 onwards, the process is clearly out-of-control. Similarly, the values of $Z_{i}^{*}$ and the control limit of upward EWMA- $\gamma^{2}$ are plotted in Figure 5. This figure confirms that from sample \#11 onwards, the process is clearly outof-control. Finally, the values of $S_{i}^{*}$ and the control limits of that two-sided EWMA- $\gamma^{2}$ are plotted in Figure 6. This figure confirms that from sample \#11 onwards, the process is clearly out-of-control. This once again confirms the effectiveness of the application of EWMA type control charts to monitor production processes.

\section{Concluding remarks}

In this paper, we have studied the effects of measurement errors on the performance of the Shewhart-CV control chart using a linear covariate error model. We have evaluated the performance of the Shewhart-CV control using the $A R L$ as a performance metric. We have found that the performance of the Shewhart-CV control chart in the presence of measurement errors is influenced by the precision error, i.e., the smaller the value of the precision error is, the faster the Shewhart-CV control chart is in detecting the out-ofcontrol condition. But, the precision error does not significantly affect the performance of the Shewhart-CV control chart with a normal measurement system. The performance of the Shewhart-CV control chart is influenced by the accuracy error, measured by $\theta$ and by the value of $B$. Furthermore, the performance of one-sided and two-sided EWMA- $\gamma^{2}$ control charts are influenced by measurement errors with a similar behaviour as for the Shewhart-CV control chart discussed earlier. 
Future research about control charts monitoring the coefficient of variation should be focused on the investigation of their Phase I implementation. We are currently looking into this issue and hope to report the findings in a future paper.

\section{Acknowledgements}

The authors thank the anonymous referees for their valuable suggestions which helped to improve the quality of the final manuscript.

\section{References}

[1] C.W. Kang, M.S. Lee, Y.J. Seong, and D.M. Hawkins. A control chart for the coefficient of variation. Journal of Quality Technology, 39(2):151-158, 2007.

[2] P. Castagliola, G. Celano, and S. Psarakis. Monitoring the coefficient of variation using EWMA charts. Journal of Quality Technology, 43(3):249-265, 2011.

[3] M.E. Calzada and S. M. Scariano. A synthetic control chart for the coefficient of variation. Journal of Statistical Computation and Simulation, 83(5):853-867, 2013.

[4] P. Castagliola, A. Achouri, H. Taleb, G. Celano, and S. Psarakis. Monitoring the coefficient of variation using a variable sampling interval control chart. Quality and Reliability Engineering International, 29(8):1135-1149, 2013.

[5] P. Castagliola, A. Achouri, H. Taleb, G. Celano, and S. Psarakis. Monitoring the coefficient of variation using control charts with run rules. Quality Technology and Quantitative Management, 10(1):75-94, 2013.

[6] J. Zhang, Z. Li, B. Chen, and Z. Wang. A new exponentially weighted moving average control chart for monitoring the coefficient of variation. Computers $\mathcal{E}$ Industrial Engineering, 78:205-212, 2014.

[7] P. Castagliola, A. Amdouni, H. Taleb, and G. Celano. One-sided Shewhart-type charts for monitoring the coefficient of variation in short production runs. Quality Technology and Quantitative Management, 12(1):53-67, 2015.

[8] P. Castagliola, A. Achouri, H. Taleb, G. Celano, and S. Psarakis. Monitoring the coefficient of variation using a variable sample size control chart. The International Journal of Advanced Manufacturing Technology, 81(9-12):1561-1576, 2015.

[9] A. Amdouni, P. Castagliola, H. Taleb, and G. Celano. Monitoring the coefficient of variation using a variable sample size control chart in short production runs. The International Journal of Advanced Manufacturing Technology, 81(1-4):1-14, 2015.

[10] H.W. You, B.C.M. Michael Khoo, P. Castagliola, and A. Haq. Monitoring the coefficient of variation using the side sensitive group runs chart. Quality and Reliability Engineering International, 32(5):1913-1927, 2016. 
[11] P.H. Tran and K. P. Tran. The efficiency of CUSUM schemes for monitoring the coefficient of variation. Stochastic Models in Business and Industry, 32(6):870-881, 2016.

[12] C.A. Bennet. Effect of measurement error on chemical process control. Industry Quality Control, 10(4):17-20, 1954.

[13] T. Kanazuka. The effect of measurement error on the power of the $\bar{X}$ and $R$ charts. Journal of Quality Technology, 18(2):91-95, 1986.

[14] K.W. Linna and W.H. Woodall. Effect of measurement error on Shewhart control chart. Journal of Quality Technology, 33(2):213-222, 2001.

[15] K.W. Linna, W.H. Woodall, and K.L. Busby. The performance of multivariate control charts in the presence of measurement error. Journal of Quality Technology, 33(3):349, 2001.

[16] P.E. Maravelakis. EWMA chart and measurement error. Journal of Applied Statistics, 31(4):445-455, 2004.

[17] A.F.B. Costa and P. Castagliola. Effect of measurement error and autocorrelation on the $\bar{X}$ chart. Journal of Applied Statistics, 38(4):661-673, 2011.

[18] P.E. Maravelakis. Measurement error effect on the CUSUM control chart. Journal of Applied Statistics, 39(2):323-336, 2012.

[19] X.L. Hu, P. Castagliola, J. Sun, and M.B.C. Khoo. The effect of measurement errors on the synthetic $\bar{X}$ chart. Quality and Reliability Engineering International, 31(8):1769-1778, 2015.

[20] R. Noorossana and Y. Zerehsaz. Effect of measurement error on phase II monitoring of simple linear profiles. The International Journal of Advanced Manufacturing Technology, 79(9-12):2031-2040, 2015.

[21] K.P. Tran, P. Castagliola, and G. Celano. The performance of the Shewhart-RZ control chart in the presence of measurement error. International Journal of Production Research, 54:7504-7522, 2016.

[22] K.P. Tran, P. Castagliola, and N. Balakrishnan. On the performance of Shewhart median chart in the presence of measurement errors. Quality and Reliability Engineering International, 33(5):1019-1029, 2017.

[23] K.P. Tran. Run rules median control charts for monitoring process mean in manufacturing. Quality and Reliability Engineering International, 2017. In press, DOI: $10.1002 /$ qre.2201.

[24] W.C. Yeong, M.B.C. Khoo, S.L. Lim, and W.L. Teoh. The coefficient of variation chart with measurement error. Quality Technology \& Quantitative Management, pages $1-25,2017$. 
[25] A.T. McKay. Distribution of the coefficient of variation and extended $t$ distribution. Journal of the Royal Statistical Society, 95:695-698, 1932.

[26] W.A. Hendricks and W.K. Robey. The sampling distribution of the coefficient of variation. Annals of Mathematical Statistics, 7:129-132, 1936.

[27] B. Iglewicz, R.H. Myers, and R.B. Howe. On the percentage points of the sample coefficient of variation. Biometrika, 55(3):580-581, 1968.

[28] B. Iglewicz and R.H. Myers. Comparisons of approximations to the percentage points of the sample coefficient of variation. Technometrics, 12(1):166-169, 1970.

[29] W.G. Warren. On the adequacy of the chi-squared approximation for the coefficient of variation. Communications in Statistics - Simulation and Computation, 11:659666,1982 .

[30] M.G. Vangel. Confidence intervals for a normal coefficient of variation. American Statistician, 15:21-26, 1996.

[31] W. Reh and B. Scheffler. Significance tests and confidence intervals for coefficients of variation. Computational Statistics \& Data Analysis, 22(4):449-452, 1996.

[32] T. Li and Q. Vuong. Nonparametric estimation of the measurement error model using multiple indicators. Journal of Multivariate Analysis, 65(2):139-165, 1998.

[33] D.C. Montgomery. Statistical Quality Control: a Modern Introduction, 7th Edn. John Wiley \& Sons,Hoboken, New Jersey, 2013.

[34] R. Breunig. An almost unbiased estimator of the coefficient of variation. Economics Letters, 70(1):15-19, 2001.

[35] D. Brook and D.A. Evans. An approach to the probability distribution of CUSUM run length. Biometrika, 59(3):539-549, 1972.

[36] A. Chen and Y. K. Chen. Design of EWMA and CUSUM control charts subject to random shift sizes and quality impacts. IIE Transactions, 39(12):1127-1141, 2007.

[37] G. Celano, P. Castagliola, G. Nenes, and S. Fichera. Performance of $t$ control charts in short runs with unknown shift sizes. Computers $\mathcal{E}$ Industrial Engineering, 64: $56-68,2013$. 


\begin{tabular}{|c|c|c|c|c|c|c|}
\hline$\eta$ & $\theta$ & $\gamma_{0}$ & $n=5$ & $n=7$ & $n=10$ & $n=15$ \\
\hline \multicolumn{7}{|c|}{$B=1$} \\
\hline \multirow[t]{8}{*}{0.10} & 0.01 & 0.05 & 0.0081 & 0.0132 & 0.0185 & 0.0238 \\
\hline & & & 0.1053 & 0.0950 & 0.0865 & 0.0791 \\
\hline & & 0.10 & 0.0161 & 0.0263 & 0.0368 & 0.0475 \\
\hline & & & 0.2130 & 0.1917 & 0.1743 & 0.1591 \\
\hline & & 0.15 & 0.0241 & 0.0394 & 0.0550 & 0.0709 \\
\hline & & & 0.3257 & 0.2919 & 0.2646 & 0.2408 \\
\hline & & 0.20 & 0.0320 & 0.0522 & 0.0729 & 0.0940 \\
\hline & & & 0.4464 & 0.3978 & 0.3589 & 0.3253 \\
\hline \multirow{8}{*}{0.28} & 0.05 & 0.05 & 0.0080 & 0.0131 & 0.0183 & 0.0236 \\
\hline & & & 0.1047 & 0.0944 & 0.0860 & 0.0786 \\
\hline & & 0.10 & 0.0160 & 0.0262 & 0.0366 & 0.0472 \\
\hline & & & 0.2117 & 0.1905 & 0.1732 & 0.1581 \\
\hline & & 0.15 & 0.0240 & 0.0391 & 0.0547 & 0.0705 \\
\hline & & & 0.3236 & 0.2901 & 0.2629 & 0.2393 \\
\hline & & 0.20 & 0.0318 & 0.0519 & 0.0725 & 0.0935 \\
\hline & & & 0.4434 & 0.3952 & 0.3565 & 0.3232 \\
\hline \multicolumn{7}{|c|}{$B=5$} \\
\hline \multirow[t]{8}{*}{0.10} & 0.01 & 0.05 & 0.0081 & 0.0132 & 0.0185 & 0.0239 \\
\hline & & & 0.1057 & 0.0953 & 0.0868 & 0.0793 \\
\hline & & 0.10 & 0.0162 & 0.0264 & 0.0369 & 0.0476 \\
\hline & & & 0.2137 & 0.1923 & 0.1749 & 0.1596 \\
\hline & & 0.15 & 0.0242 & 0.0395 & 0.0552 & 0.0711 \\
\hline & & & 0.3268 & 0.2929 & 0.2655 & 0.2416 \\
\hline & & 0.20 & 0.0321 & 0.0524 & 0.0732 & 0.0943 \\
\hline & & & 0.4480 & 0.3992 & 0.3601 & 0.3264 \\
\hline \multirow[t]{8}{*}{0.28} & 0.05 & 0.05 & 0.0081 & 0.0132 & 0.0184 & 0.0237 \\
\hline & & & 0.1050 & 0.0947 & 0.0862 & 0.0788 \\
\hline & & 0.10 & 0.0161 & 0.0263 & 0.0367 & 0.0473 \\
\hline & & & 0.2123 & 0.1910 & 0.1737 & 0.1585 \\
\hline & & 0.15 & 0.0240 & 0.0392 & 0.0548 & 0.0707 \\
\hline & & & 0.3245 & 0.2909 & 0.2637 & 0.2400 \\
\hline & & 0.20 & 0.0319 & 0.0520 & 0.0727 & 0.0937 \\
\hline & & & 0.4447 & 0.3963 & 0.3576 & 0.3241 \\
\hline
\end{tabular}

Table 1: Values of $L C L$ (first row) and $U C L$ (second row) for the Shewhart-CV control chart in the presence of measurement errors, for different values of $\eta, \theta, n, \gamma_{0}, B$ and $m=1$. 


\begin{tabular}{|c|c|c|c|c|c|c|}
\hline \multirow[b]{2}{*}{$\tau$} & \multicolumn{6}{|c|}{$n=5$} \\
\hline & $\eta=0$ & $\eta=0.1$ & $\eta=0.2$ & $\eta=0.3$ & $\eta=0.5$ & $\eta=1$ \\
\hline 0.5 & $(56.34,56.66,57.91)$ & $(56.34,56.66,57.93)$ & $(56.34,56.67,57.98)$ & $(56.35,56.69,58.06)$ & $(56.37,56.76,58.32)$ & $(56.44,57.07,59.56)$ \\
\hline 0.7 & $(148.13,148.80,151.37)$ & $(148.14,148.81,151.41)$ & $(148.14,148.83,151.51)$ & $(148.15,148.88,151.68)$ & $(148.19,149.02,152.22)$ & $(148.36,149.67,154.72)$ \\
\hline 0.8 & $(319.58,320.48,323.77)$ & $(319.58,320.49,323.82)$ & $(319.60,320.52,323.94)$ & $(319.61,320.59,324.16)$ & $(319.66,320.76,324.84)$ & $(319.90,321.60,327.93)$ \\
\hline 1.3 & $(48.70,49.20,51.32)$ & $(48.70,49.21,51.35)$ & $(48.71,49.23,51.44)$ & $(48.72,49.26,51.59)$ & $(48.74,49.37,52.07)$ & $(48.86,49.89,54.42)$ \\
\hline 1.5 & $(12.30,12.49,13.32)$ & $(12.30,12.50,13.33)$ & $(12.30,12.50,13.36)$ & $(12.31,12.52,13.42)$ & $(12.32,12.56,13.61)$ & $(12.37,12.76,14.55)$ \\
\hline \multirow[t]{2}{*}{2.0} & $(3.36,3.42,3.70)$ & $(3.36,3.42,3.70)$ & $(3.36,3.43,3.71)$ & $(3.36,3.43,3.73)$ & $(3.37,3.45,3.79)$ & $(3.38,3.51,4.10)$ \\
\hline & & & $n=7$ & & & \\
\hline$\tau$ & $\eta=0$ & $\eta=0.1$ & $\eta=0.2$ & $\eta=0.3$ & $\eta=0.5$ & $\eta=1$ \\
\hline 0.5 & $(20.71,20.89,21.61)$ & $(20.71,20.89,21.62)$ & $(20.72,20.90,21.64)$ & $(20.72,20.91,21.69)$ & $(20.73,20.95,21.84)$ & $(20.77,21.13,22.55)$ \\
\hline 0.7 & $(75.72,76.27,78.42)$ & $(75.72,76.27,78.45)$ & $(75.72,76.30,78.54)$ & $(75.73,76.33,78.68)$ & $(75.76,76.45,79.13)$ & $(75.90,76.99,81.24)$ \\
\hline 0.8 & $(223.38,224.43,228.42)$ & $(223.38,224.44,228.48)$ & $(223.40,224.49,228.63)$ & $(223.41,224.56,228.89)$ & $(223.47,224.77,229.72)$ & $(223.74,225.78,233.50)$ \\
\hline 1.3 & $(36.67,37.16,39.22)$ & $(36.67,37.17,39.25)$ & $(36.68,37.19,39.34)$ & $(36.68,37.22,39.48)$ & $(36.71,37.33,39.94)$ & $(36.83,37.83,42.18)$ \\
\hline 1.5 & $(8.42,8.58,9.27)$ & $(8.42,8.58,9.28)$ & $(8.42,8.59,9.30)$ & $(8.43,8.60,9.35)$ & $(8.43,8.64,9.51)$ & $(8.47,8.80,10.26)$ \\
\hline \multirow[t]{2}{*}{2.0} & $(2.36,2.41,2.61)$ & $(2.36,2.41,2.61)$ & $(2.36,2.41,2.62)$ & $(2.36,2.41,2.63)$ & $(2.36,2.42,2.68)$ & $(2.37,2.47,2.91)$ \\
\hline & & & $n=10$ & & & \\
\hline$\tau$ & $\eta=0$ & $\eta=0.1$ & $\eta=0.2$ & $\eta=0.3$ & $\eta=0.5$ & $\eta=1$ \\
\hline 0.5 & $(7.02,7.10,7.41)$ & $(7.02,7.10,7.41)$ & $(7.02,7.10,7.42)$ & $(7.02,7.11,7.44)$ & $(7.03,7.12,7.51)$ & $(7.05,7.20,7.82)$ \\
\hline 0.7 & $(33.76,34.11,35.49)$ & $(33.76,34.11,35.51)$ & $(33.76,34.12,35.57)$ & $(33.77,34.15,35.66)$ & $(33.78,34.22,35.95)$ & $(33.87,34.57,37.32)$ \\
\hline 0.8 & $(141.35,142.36,146.24)$ & $(141.36,142.37,146.29)$ & $(141.37,142.41,146.44)$ & $(141.39,142.48,146.70)$ & $(141.44,142.69,147.50)$ & $(141.69,143.67,151.20)$ \\
\hline 1.3 & $(26.22,26.65,28.44)$ & $(26.22,26.65,28.46)$ & $(26.23,26.67,28.54)$ & $(26.23,26.70,28.66)$ & $(26.26,26.79,29.05)$ & $(26.36,27.23,30.96)$ \\
\hline 1.5 & $(5.58,5.70,6.21)$ & $(5.58,5.70,6.21)$ & $(5.58,5.71,6.23)$ & $(5.58,5.71,6.27)$ & $(5.59,5.74,6.38)$ & $(5.62,5.86,6.93)$ \\
\hline \multirow[t]{2}{*}{2.0} & $(1.70,1.73,1.87)$ & $(1.70,1.73,1.87)$ & $(1.70,1.73,1.87)$ & $(1.70,1.74,1.88)$ & $(1.70,1.74,1.91)$ & $(1.71,1.78,2.06)$ \\
\hline & & & $n=15$ & & & \\
\hline$\tau$ & $\eta=0$ & $\eta=0.1$ & $\eta=0.2$ & $\eta=0.3$ & $\eta=0.5$ & $\eta=1$ \\
\hline 0.5 & $(2.44,2.46,2.57)$ & $(2.44,2.46,2.57)$ & $(2.44,2.47,2.57)$ & $(2.44,2.47,2.58)$ & $(2.44,2.47,2.60)$ & $(2.45,2.50,2.70)$ \\
\hline 0.7 & $(12.70,12.87,13.54)$ & $(12.70,12.87,13.55)$ & $(12.70,12.88,13.58)$ & $(12.71,12.89,13.63)$ & $(12.72,12.93,13.77)$ & $(12.76,13.09,14.45)$ \\
\hline 0.8 & $(76.97,77.75,80.80)$ & $(76.98,77.76,80.83)$ & $(76.98,77.79,80.96)$ & $(77.00,77.84,81.15)$ & $(77.04,78.01,81.79)$ & $(77.23,78.78,84.74)$ \\
\hline 1.3 & $(17.13,17.46,18.82)$ & $(17.14,17.47,18.84)$ & $(17.14,17.48,18.90)$ & $(17.14,17.50,18.99)$ & $(17.16,17.57,19.29)$ & $(17.24,17.91,20.74)$ \\
\hline 1.5 & $(3.50,3.57,3.90)$ & $(3.50,3.57,3.90)$ & $(3.50,3.58,3.92)$ & $(3.50,3.58,3.94)$ & $(3.50,3.60,4.01)$ & $(3.52,3.68,4.37)$ \\
\hline 2.0 & $(1.28,1.30,1.37)$ & $(1.28,1.30,1.37)$ & $(1.28,1.30,1.37)$ & $(1.28,1.30,1.38)$ & $(1.28,1.30,1.40)$ & $(1.28,1.32,1.48)$ \\
\hline
\end{tabular}

Table 2: $A R L$ values of Shewhart-CV control chart in the presence of measurement errors for $\gamma_{0}=0.05$ (left side), $\gamma_{0}=0.1$ (middle) and $\gamma_{0}=0.2$ (right side), for different values of $\eta, \theta=0.05, \tau, n, B=1, m=1$. 


\begin{tabular}{|c|c|c|c|c|c|c|}
\hline \multirow[b]{2}{*}{$\tau$} & \multicolumn{5}{|c|}{$n=5$} & \multirow[b]{2}{*}{$\theta=0.05$} \\
\hline & $\theta=0$ & $\theta=0.01$ & $\theta=0.02$ & $\theta=0.03$ & $\theta=0.04$ & \\
\hline 0.5 & $(51.52,51.86,53.24)$ & $(52.48,52.82,54.20)$ & $(53.44,53.78,55.15)$ & $(54.40,54.75,56.11)$ & $(55.37,55.72,57.07)$ & $(56.35,56.69,58.04)$ \\
\hline 0.7 & $(138.93,139.68,142.62)$ & $(140.78,141.53,144.43)$ & $(142.63,143.38,146.24)$ & $(144.48,145.21,148.05)$ & $(146.32,147.04,149.85)$ & $(148.15,148.87,151.64)$ \\
\hline 0.8 & $(308.39,309.45,313.38)$ & $(310.70,311.73,315.58)$ & $(312.97,313.98,317.75)$ & $(315.20,316.21,319.89)$ & $(317.42,318.39,322.02)$ & $(319.61,320.57,324.11)$ \\
\hline 1.3 & $(43.58,44.14,46.53)$ & $(44.60,45.15,47.53)$ & $(45.62,46.17,48.53)$ & $(46.65,47.19,49.53)$ & $(47.68,48.22,50.54)$ & $(48.71,49.25,51.55)$ \\
\hline 1.5 & $(10.58,10.78,11.66)$ & $(10.91,11.12,12.00)$ & $(11.25,11.46,12.34)$ & $(11.60,11.80,12.69)$ & $(11.95,12.16,13.05)$ & $(12.31,12.51,13.41)$ \\
\hline \multirow[t]{2}{*}{2.0} & $(2.89,2.96,3.24)$ & $(2.98,3.05,3.33)$ & $(3.07,3.14,3.43)$ & $(3.17,3.23,3.52)$ & $(3.26,3.33,3.62)$ & $(3.36,3.43,3.73)$ \\
\hline & & & $n=7$ & & & \\
\hline$\tau$ & $\theta=0$ & $\theta=0.01$ & $\theta=0.02$ & $\theta=0.03$ & $\theta=0.04$ & $\theta=0.05$ \\
\hline 0.5 & $(18.45,18.64,19.40)$ & $(18.90,19.09,19.85)$ & $(19.34,19.54,20.30)$ & $(19.80,19.99,20.76)$ & $(20.26,20.45,21.22)$ & $(20.72,20.91,21.68)$ \\
\hline 0.7 & $(69.32,69.93,72.32)$ & $(70.60,71.20,73.58)$ & $(71.88,72.48,74.84)$ & $(73.16,73.76,76.11)$ & $(74.44,75.04,77.38)$ & $(75.73,76.32,78.65)$ \\
\hline 0.8 & $(212.10,213.32,217.97)$ & $(214.39,215.59,220.17)$ & $(216.67,217.86,222.36)$ & $(218.93,220.10,224.53)$ & $(221.18,222.33,226.69)$ & $(223.41,224.54,228.83)$ \\
\hline 1.3 & $(32.45,32.99,35.28)$ & $(33.28,33.82,36.10)$ & $(34.12,34.66,36.93)$ & $(34.97,35.50,37.76)$ & $(35.82,36.35,38.60)$ & $(36.68,37.21,39.45)$ \\
\hline 1.5 & $(7.21,7.38,8.10)$ & $(7.45,7.61,8.34)$ & $(7.68,7.85,8.58)$ & $(7.93,8.10,8.83)$ & $(8.17,8.35,9.08)$ & $(8.43,8.60,9.34)$ \\
\hline \multirow[t]{2}{*}{2.0} & $(2.06,2.11,2.32)$ & $(2.12,2.17,2.38)$ & $(2.17,2.22,2.44)$ & $(2.23,2.28,2.50)$ & $(2.30,2.35,2.56)$ & $(2.36,2.41,2.63)$ \\
\hline & & & $n=10$ & & & \\
\hline$\tau$ & $\theta=0$ & $\theta=0.01$ & $\theta=0.02$ & $\theta=0.03$ & $\theta=0.04$ & $\theta=0.05$ \\
\hline 0.5 & $(6.17,6.25,6.57)$ & $(6.34,6.42,6.74)$ & $(6.51,6.59,6.91)$ & $(6.68,6.76,7.08)$ & $(6.85,6.93,7.26)$ & $(7.02,7.11,7.44)$ \\
\hline 0.7 & $(30.16,30.54,32.03)$ & $(30.87,31.25,32.74)$ & $(31.58,31.96,33.46)$ & $(32.30,32.68,34.18)$ & $(33.03,33.41,34.90)$ & $(33.76,34.14,35.64)$ \\
\hline 0.8 & $(131.70,132.84,137.26)$ & $(133.64,134.77,139.14)$ & $(135.59,136.71,141.02)$ & $(137.52,138.63,142.90)$ & $(139.46,140.55,144.77)$ & $(141.38,142.46,146.64)$ \\
\hline 1.3 & $(22.96,23.42,25.37)$ & $(23.60,24.06,26.01)$ & $(24.25,24.71,26.65)$ & $(24.90,25.36,27.30)$ & $(25.56,26.02,27.96)$ & $(26.23,26.69,28.63)$ \\
\hline 1.5 & $(4.78,4.90,5.43)$ & $(4.93,5.06,5.59)$ & $(5.09,5.22,5.75)$ & $(5.25,5.38,5.92)$ & $(5.42,5.54,6.09)$ & $(5.58,5.71,6.26)$ \\
\hline \multirow[t]{2}{*}{2.0} & $(1.52,1.56,1.69)$ & $(1.56,1.59,1.73)$ & $(1.59,1.62,1.76)$ & $(1.63,1.66,1.80)$ & $(1.66,1.70,1.84)$ & $(1.70,1.74,1.88)$ \\
\hline & & & $n=15$ & & & \\
\hline$\tau$ & $\theta=0$ & $\theta=0.01$ & $\theta=0.02$ & $\theta=0.03$ & $\theta=0.04$ & $\theta=0.05$ \\
\hline 0.5 & $(2.18,2.21,2.31)$ & $(2.23,2.26,2.36)$ & $(2.28,2.31,2.41)$ & $(2.33,2.36,2.47)$ & $(2.39,2.41,2.52)$ & $(2.44,2.47,2.58)$ \\
\hline 0.7 & $(11.13,11.30,12.01)$ & $(11.43,11.61,12.32)$ & $(11.74,11.92,12.64)$ & $(12.06,12.24,12.96)$ & $(12.38,12.56,13.28)$ & $(12.71,12.89,13.62)$ \\
\hline 0.8 & $(70.11,70.98,74.35)$ & $(71.48,72.34,75.70)$ & $(72.85,73.71,77.04)$ & $(74.23,75.08,78.40)$ & $(75.61,76.45,79.75)$ & $(76.99,77.83,81.11)$ \\
\hline 1.3 & $(14.85,15.20,16.65)$ & $(15.29,15.64,17.10)$ & $(15.74,16.10,17.56)$ & $(16.20,16.56,18.02)$ & $(16.67,17.02,18.49)$ & $(17.14,17.50,18.97)$ \\
\hline 1.5 & $(3.02,3.10,3.43)$ & $(3.11,3.19,3.53)$ & $(3.20,3.28,3.63)$ & $(3.30,3.38,3.73)$ & $(3.40,3.48,3.83)$ & $(3.50,3.58,3.93)$ \\
\hline 2.0 & $(1.19,1.21,1.28)$ & $(1.21,1.22,1.30)$ & $(1.22,1.24,1.32)$ & $(1.24,1.26,1.34)$ & $(1.26,1.28,1.36)$ & $(1.28,1.30,1.38)$ \\
\hline
\end{tabular}

Table 3: $A R L$ values of Shewhart-CV control chart in the presence of measurement errors for $\gamma_{0}=0.05$ (left side), $\gamma_{0}=0.1$ (middle) and $\gamma_{0}=0.2$ (right side), for different values of $\theta, \eta=0.28, \tau, n, B=1, m=1$. 


\begin{tabular}{|c|c|c|c|c|c|}
\hline \multirow[b]{2}{*}{$\tau$} & \multicolumn{5}{|c|}{$n=5$} \\
\hline & $B=1$ & $B=2$ & $B=3$ & $B=4$ & $B=5$ \\
\hline 0.5 & $(56.35,56.69,58.04)$ & $(53.91,54.24,55.53)$ & $(53.11,53.43,54.71)$ & $(52.71,53.03,54.31)$ & $(52.47,52.79,54.07)$ \\
\hline 0.7 & $(148.15,148.87,151.64)$ & $(143.54,144.24,146.94)$ & $(142.00,142.70,145.39)$ & $(141.23,141.93,144.62)$ & $(140.77,141.46,144.16)$ \\
\hline 0.8 & $(319.61,320.57,324.11)$ & $(314.07,315.03,318.56)$ & $(312.18,313.15,316.71)$ & $(311.24,312.21,315.78)$ & $(310.67,311.64,315.23)$ \\
\hline 1.3 & $(48.71,49.25,51.55)$ & $(46.12,46.64,48.85)$ & $(45.27,45.78,47.98)$ & $(44.84,45.35,47.55)$ & $(44.58,45.10,47.30)$ \\
\hline 1.5 & $(12.31,12.51,13.41)$ & $(11.42,11.62,12.45)$ & $(11.13,11.33,12.15)$ & $(10.99,11.18,12.00)$ & $(10.91,11.10,11.91)$ \\
\hline \multirow[t]{2}{*}{2.0} & $(3.36,3.43,3.73)$ & $(3.12,3.18,3.45)$ & $(3.04,3.10,3.37)$ & $(3.00,3.06,3.33)$ & $(2.98,3.04,3.30)$ \\
\hline & \multicolumn{5}{|c|}{$n=7$} \\
\hline$\tau$ & $B=1$ & $B=2$ & $B=3$ & $B=4$ & $B=5$ \\
\hline 0.5 & $(20.72,20.91,21.68)$ & $(19.57,19.75,20.47)$ & $(19.19,19.37,20.08)$ & $(19.00,19.18,19.89)$ & $(18.89,19.07,19.78)$ \\
\hline 0.7 & $(75.73,76.32,78.65)$ & $(72.51,73.08,75.31)$ & $(71.44,72.00,74.22)$ & $(70.90,71.47,73.68)$ & $(70.58,71.15,73.36)$ \\
\hline 0.8 & $(223.41,224.54,228.83)$ & $(217.78,218.90,223.13)$ & $(215.89,217.00,221.25)$ & $(214.93,216.06,220.32)$ & $(214.36,215.49,219.76)$ \\
\hline 1.3 & $(36.68,37.21,39.45)$ & $(34.54,35.04,37.17)$ & $(33.83,34.33,36.45)$ & $(33.48,33.98,36.09)$ & $(33.27,33.77,35.88)$ \\
\hline 1.5 & $(8.43,8.60,9.34)$ & $(7.80,7.96,8.65)$ & $(7.60,7.76,8.44)$ & $(7.50,7.66,8.33)$ & $(7.44,7.60,8.27)$ \\
\hline \multirow[t]{2}{*}{2.0} & $(2.36,2.41,2.63)$ & $(2.20,2.25,2.45)$ & $(2.15,2.20,2.40)$ & $(2.13,2.18,2.37)$ & $(2.11,2.16,2.36)$ \\
\hline & \multicolumn{5}{|c|}{$n=10$} \\
\hline$\tau$ & $B=1$ & $B=2$ & $B=3$ & $B=4$ & $B=5$ \\
\hline 0.5 & $(7.02,7.11,7.44)$ & $(6.59,6.66,6.97)$ & $(6.45,6.52,6.82)$ & $(6.38,6.45,6.75)$ & $(6.34,6.41,6.71)$ \\
\hline 0.7 & $(33.76,34.14,35.64)$ & $(31.94,32.29,33.71)$ & $(31.34,31.69,33.09)$ & $(31.04,31.39,32.78)$ & $(30.86,31.21,32.60)$ \\
\hline 0.8 & $(141.38,142.46,146.64)$ & $(136.53,137.59,141.65)$ & $(134.91,135.97,140.03)$ & $(134.10,135.16,139.22)$ & $(133.62,134.67,138.74)$ \\
\hline 1.3 & $(26.23,26.69,28.63)$ & $(24.56,25.00,26.83)$ & $(24.02,24.45,26.26)$ & $(23.75,24.18,25.99)$ & $(23.59,24.02,25.82)$ \\
\hline 1.5 & $(5.58,5.71,6.26)$ & $(5.17,5.29,5.79)$ & $(5.04,5.15,5.65)$ & $(4.97,5.09,5.58)$ & $(4.93,5.05,5.54)$ \\
\hline \multirow[t]{2}{*}{2.0} & $(1.70,1.74,1.88)$ & $(1.61,1.64,1.77)$ & $(1.58,1.61,1.74)$ & $(1.56,1.60,1.72)$ & $(1.56,1.59,1.71)$ \\
\hline & \multicolumn{5}{|c|}{$n=15$} \\
\hline$\tau$ & $B=1$ & $B=2$ & $B=3$ & $B=4$ & $B=5$ \\
\hline 0.5 & $(2.44,2.47,2.58)$ & $(2.31,2.33,2.43)$ & $(2.27,2.29,2.39)$ & $(2.24,2.27,2.37)$ & $(2.23,2.26,2.35)$ \\
\hline 0.7 & $(12.71,12.89,13.62)$ & $(11.90,12.07,12.74)$ & $(11.64,11.80,12.47)$ & $(11.51,11.67,12.33)$ & $(11.43,11.59,12.25)$ \\
\hline 0.8 & $(76.99,77.83,81.11)$ & $(73.53,74.33,77.48)$ & $(72.38,73.18,76.31)$ & $(71.80,72.60,75.73)$ & $(71.46,72.26,75.39)$ \\
\hline 1.3 & $(17.14,17.50,18.97)$ & $(15.97,16.30,17.68)$ & $(15.59,15.91,17.28)$ & $(15.40,15.72,17.08)$ & $(15.28,15.61,16.96)$ \\
\hline 1.5 & $(3.50,3.58,3.93)$ & $(3.25,3.33,3.65)$ & $(3.17,3.25,3.56)$ & $(3.13,3.21,3.52)$ & $(3.11,3.18,3.50)$ \\
\hline 2.0 & $(1.28,1.30,1.38)$ & $(1.23,1.25,1.32)$ & $(1.22,1.23,1.30)$ & $(1.21,1.23,1.30)$ & $(1.21,1.22,1.29)$ \\
\hline
\end{tabular}

Table 4: $A R L$ values of Shewhart-CV control chart in the presence of measurement errors for $\gamma_{0}=0.05$ (left side), $\gamma_{0}=0.1$ (middle) and $\gamma_{0}=0.2$ (right side), for different values of $B, \tau, n, \eta=0.28, \theta=0.01, m=1$. 


\begin{tabular}{|c|c|c|c|c|c|}
\hline \multirow[b]{2}{*}{$\tau$} & \multicolumn{5}{|c|}{$n=5$} \\
\hline & $m=1$ & $m=3$ & $m=5$ & $m=7$ & $m=10$ \\
\hline 0.5 & $(56.35,56.69,58.04)$ & $(56.34,56.67,57.95)$ & $(56.34,56.66,57.94)$ & $(56.34,56.66,57.93)$ & $(56.34,56.66,57.92)$ \\
\hline 0.7 & $(148.15,148.87,151.64)$ & $(148.14,148.82,151.46)$ & $(148.14,148.81,151.43)$ & $(148.14,148.81,151.41)$ & $(148.14,148.81,151.40)$ \\
\hline 0.8 & $(319.61,320.57,324.11)$ & $(319.59,320.51,323.89)$ & $(319.59,320.50,323.85)$ & $(319.58,320.49,323.82)$ & $(319.59,320.49,323.81)$ \\
\hline 1.3 & $(48.71,49.25,51.55)$ & $(48.71,49.22,51.40)$ & $(48.71,49.21,51.37)$ & $(48.70,49.21,51.35)$ & $(48.71,49.20,51.34)$ \\
\hline 1.5 & $(12.31,12.51,13.41)$ & $(12.30,12.50,13.35)$ & $(12.30,12.50,13.34)$ & $(12.30,12.50,13.33)$ & $(12.30,12.50,13.33)$ \\
\hline \multirow[t]{2}{*}{2.0} & $(3.36,3.43,3.73)$ & $(3.36,3.43,3.71)$ & $(3.36,3.42,3.70)$ & $(3.36,3.42,3.70)$ & $(3.36,3.42,3.70)$ \\
\hline & \multicolumn{5}{|c|}{$n=7$} \\
\hline$\tau$ & $m=1$ & $m=3$ & $m=5$ & $m=7$ & $m=10$ \\
\hline 0.5 & $(20.72,20.91,21.68)$ & $(20.71,20.90,21.63)$ & $(20.71,20.90,21.62)$ & $(20.71,20.89,21.62)$ & $(20.71,20.89,21.61)$ \\
\hline 0.7 & $(75.73,76.32,78.65)$ & $(75.72,76.29,78.50)$ & $(75.72,76.28,78.47)$ & $(75.72,76.27,78.46)$ & $(75.72,76.27,78.45)$ \\
\hline 0.8 & $(223.41,224.54,228.83)$ & $(223.39,224.47,228.56)$ & $(223.39,224.45,228.51)$ & $(223.38,224.45,228.49)$ & $(223.38,224.44,228.46)$ \\
\hline 1.3 & $(36.68,37.21,39.45)$ & $(36.67,37.18,39.30)$ & $(36.67,37.17,39.27)$ & $(36.67,37.17,39.26)$ & $(36.67,37.16,39.25)$ \\
\hline 1.5 & $(8.43,8.60,9.34)$ & $(8.42,8.59,9.29)$ & $(8.42,8.59,9.28)$ & $(8.42,8.58,9.28)$ & $(8.42,8.58,9.27)$ \\
\hline \multirow[t]{2}{*}{2.0} & $(2.36,2.41,2.63)$ & $(2.36,2.41,2.62)$ & $(2.36,2.41,2.61)$ & $(2.36,2.41,2.61)$ & $(2.36,2.41,2.61)$ \\
\hline & \multicolumn{5}{|c|}{$n=10$} \\
\hline$\tau$ & $m=1$ & $m=3$ & $m=5$ & $m=7$ & $m=10$ \\
\hline 0.5 & $(7.02,7.11,7.44)$ & $(7.02,7.10,7.42)$ & $(7.02,7.10,7.41)$ & $(7.02,7.10,7.41)$ & $(7.02,7.10,7.41)$ \\
\hline 0.7 & $(33.76,34.14,35.64)$ & $(33.76,34.12,35.54)$ & $(33.76,34.11,35.52)$ & $(33.76,34.11,35.51)$ & $(33.76,34.11,35.51)$ \\
\hline 0.8 & $(141.38,142.46,146.64)$ & $(141.36,142.39,146.37)$ & $(141.36,142.38,146.32)$ & $(141.36,142.37,146.30)$ & $(141.36,142.37,146.28)$ \\
\hline 1.3 & $(26.23,26.69,28.63)$ & $(26.22,26.66,28.50)$ & $(26.22,26.66,28.48)$ & $(26.22,26.65,28.47)$ & $(26.22,26.65,28.46)$ \\
\hline 1.5 & $(5.58,5.71,6.26)$ & $(5.58,5.70,6.22)$ & $(5.58,5.70,6.22)$ & $(5.58,5.70,6.21)$ & $(5.58,5.70,6.21)$ \\
\hline \multirow[t]{2}{*}{2.0} & $(1.70,1.74,1.88)$ & $(1.70,1.73,1.87)$ & $(1.70,1.73,1.87)$ & $(1.70,1.73,1.87)$ & $(1.70,1.73,1.87)$ \\
\hline & \multicolumn{5}{|c|}{$n=15$} \\
\hline$\tau$ & $m=1$ & $m=3$ & $m=5$ & $m=7$ & $m=10$ \\
\hline 0.5 & $(2.44,2.47,2.58)$ & $(2.44,2.47,2.57)$ & $(2.44,2.46,2.57)$ & $(2.44,2.46,2.57)$ & $(2.44,2.46,2.57)$ \\
\hline 0.7 & $(12.71,12.89,13.62)$ & $(12.70,12.88,13.57)$ & $(12.70,12.87,13.56)$ & $(12.70,12.87,13.55)$ & $(12.70,12.87,13.55)$ \\
\hline 0.8 & $(76.99,77.83,81.11)$ & $(76.98,77.78,80.90)$ & $(76.98,77.77,80.86)$ & $(76.98,77.76,80.84)$ & $(76.97,77.76,80.83)$ \\
\hline 1.3 & $(17.14,17.50,18.97)$ & $(17.14,17.47,18.87)$ & $(17.14,17.47,18.85)$ & $(17.14,17.47,18.85)$ & $(17.14,17.46,18.84)$ \\
\hline 1.5 & $(3.50,3.58,3.93)$ & $(3.50,3.58,3.91)$ & $(3.50,3.57,3.91)$ & $(3.50,3.57,3.90)$ & $(3.50,3.57,3.90)$ \\
\hline 2.0 & $(1.28,1.30,1.38)$ & $(1.28,1.30,1.37)$ & $(1.28,1.30,1.37)$ & $(1.28,1.30,1.37)$ & $(1.28,1.30,1.37)$ \\
\hline
\end{tabular}

Table 5: $A R L$ values of Shewhart-CV control chart in the presence of measurement errors for $\gamma_{0}=0.05$ (left side), $\gamma_{0}=0.1$ (middle) and $\gamma_{0}=0.2$ (right side), for different values of $m, \tau, n, \eta=0.28, \theta=0.05, B=1$. 


\begin{tabular}{|c|c|c|c|c|c|c|}
\hline \multirow[b]{2}{*}{$\tau$} & \multicolumn{6}{|c|}{$n=5$} \\
\hline & $\eta=0$ & $\eta=0.1$ & $\eta=0.2$ & $\eta=0.3$ & $\eta=0.5$ & $\eta=1$ \\
\hline \multirow[t]{2}{*}{0.5} & $(2.6906,2.7006,2.8184)$ & $(2.6908,2.7008,2.8208)$ & $(2.6911,2.7021,2.8284)$ & $(2.6910,2.7038,2.8410)$ & $(2.6933,2.7028,2.8829)$ & $(2.6962,2.7232,3.1070)$ \\
\hline & $(0.0793,0.0745,0.0610)$ & $(0.0793,0.0744,0.0609)$ & $(0.0793,0.0744,0.0606)$ & $(0.0792,0.0742,0.0600)$ & $(0.0792,0.0726,0.0583)$ & $(0.0781,0.0686,0.0520)$ \\
\hline \multirow[t]{2}{*}{0.7} & $(2.5514,2.5496,2.6439)$ & $(2.5540,2.5491,2.6487)$ & $(2.5543,2.5495,2.6561)$ & $(2.5547,2.5468,2.6696)$ & $(2.5495,2.5523,2.7126)$ & $(2.5526,2.5589,2.9715)$ \\
\hline & $(0.0574,0.0533,0.0418)$ & $(0.0578,0.0532,0.0419)$ & $(0.0578,0.0531,0.0416)$ & $(0.0578,0.0524,0.0412)$ & $(0.0568,0.0522,0.0396)$ & $(0.0563,0.0483,0.0350)$ \\
\hline \multirow[t]{2}{*}{0.8} & $(2.2972,2.2764,2.3479)$ & $(2.2923,2.2785,2.3482)$ & $(2.2973,2.2738,2.3566)$ & $(2.2959,2.2734,2.3700)$ & $(2.2882,2.2690,2.4272)$ & $(2.2870,2.2683,2.7458)$ \\
\hline & $(0.0302,0.0277,0.0209)$ & $(0.0299,0.0278,0.0208)$ & $(0.0302,0.0274,0.0206)$ & $(0.0301,0.0273,0.0202)$ & $(0.0295,0.0267,0.0195)$ & $(0.0292,0.0247,0.0155)$ \\
\hline \multirow[t]{2}{*}{1.3} & $(2.7947,2.8061,2.9920)$ & $(2.7915,2.8061,2.9937)$ & $(2.7880,2.8080,3.0079)$ & $(2.7887,2.8106,3.0216)$ & $(2.7897,2.8146,3.0827)$ & $(2.7935,2.8498,3.3372)$ \\
\hline & $(0.0979,0.0916,0.0856)$ & $(0.0972,0.0915,0.0854)$ & $(0.0965,0.0915,0.0861)$ & $(0.0965,0.0914,0.0858)$ & $(0.0963,0.0903,0.0874)$ & $(0.0949,0.0875,0.0892)$ \\
\hline \multirow[t]{2}{*}{1.5} & $(3.1542,3.2086,3.4227)$ & $(3.1522,3.2141,3.4296)$ & $(3.1559,3.2138,3.4329)$ & $(3.1577,3.2149,3.4476)$ & $(3.1614,3.2290,3.4967)$ & $(3.1823,3.2836,3.7066)$ \\
\hline & $(0.1741,0.1748,0.1749)$ & $(0.1736,0.1760,0.1759)$ & $(0.1744,0.1754,0.1745)$ & $(0.1746,0.1748,0.1745)$ & $(0.1749,0.1755,0.1750)$ & $(0.1769,0.1760,0.1699)$ \\
\hline \multirow[t]{2}{*}{2.0} & $(3.7100,3.7559,3.9346)$ & $(3.7081,3.7529,3.9353)$ & $(3.7139,3.7545,3.9422)$ & $(3.7137,3.7624,3.9512)$ & $(3.7175,3.7738,3.9880)$ & $(3.7307,3.8094,4.1847)$ \\
\hline & $(0.3674,0.3642,0.3454)$ & $(0.3664,0.3625,0.3444)$ & $(0.3688,0.3623,0.3435)$ & $(0.3684,0.3642,0.3408)$ & $(0.3688,0.3642,0.3354)$ & $(0.3688,0.3559,0.3160)$ \\
\hline & \multicolumn{6}{|c|}{$n=7$} \\
\hline$\tau$ & $\eta=0$ & $\eta=0.1$ & $\eta=0.2$ & $\eta=0.3$ & $\eta=0.5$ & $\eta=1$ \\
\hline \multirow[t]{2}{*}{0.5} & $(2.8163,2.8279,2.9185)$ & $(2.8184,2.8284,2.9237)$ & $(2.8180,2.8304,2.9287)$ & $(2.8162,2.8313,2.9388)$ & $(2.8191,2.8366,2.9665)$ & $(2.8207,2.8523,3.1201)$ \\
\hline & $(0.1118,0.1063,0.0900)$ & $(0.1123,0.1063,0.0905)$ & $(0.1121,0.1064,0.0900)$ & $(0.1115,0.1060,0.0896)$ & $(0.1118,0.1054,0.0870)$ & $(0.1101,0.1003,0.0780)$ \\
\hline \multirow[t]{2}{*}{0.7} & $(2.6744,2.6784,2.7494)$ & $(2.6772,2.6778,2.7511)$ & $(2.6775,2.6786,2.7567)$ & $(2.6764,2.6799,2.7663)$ & $(2.6758,2.6796,2.7960)$ & $(2.6773,2.6873,2.9692)$ \\
\hline & $(0.0802,0.0758,0.0621)$ & $(0.0807,0.0756,0.0620)$ & $(0.0807,0.0755,0.0617)$ & $(0.0804,0.0754,0.0612)$ & $(0.0800,0.0742,0.0593)$ & $(0.0791,0.0698,0.0526)$ \\
\hline \multirow[t]{2}{*}{0.8} & $(2.4318,2.4203,2.4647)$ & $(2.4318,2.4205,2.4650)$ & $(2.4319,2.4195,2.4725)$ & $(2.4316,2.4183,2.4827)$ & $(2.4322,2.4164,2.5153)$ & $(2.4282,2.4166,2.7372)$ \\
\hline & $(0.0424,0.0396,0.0314)$ & $(0.0424,0.0396,0.0312)$ & $(0.0424,0.0394,0.0311)$ & $(0.0423,0.0391,0.0308)$ & $(0.0423,0.0385,0.0296)$ & $(0.0415,0.0362,0.0256)$ \\
\hline \multirow[t]{2}{*}{1.3} & $(2.8626,2.8836,3.0187)$ & $(2.8627,2.8813,3.0218)$ & $(2.8630,2.8828,3.0258)$ & $(2.8634,2.8852,3.0361)$ & $(2.8653,2.8906,3.0782)$ & $(2.8711,2.9105,3.2766)$ \\
\hline & $(0.1232,0.1191,0.1102)$ & $(0.1232,0.1184,0.1103)$ & $(0.1232,0.1184,0.1096)$ & $(0.1231,0.1184,0.1091)$ & $(0.1231,0.1176,0.1094)$ & $(0.1223,0.1127,0.1110)$ \\
\hline \multirow[t]{2}{*}{1.5} & $(3.2372,3.2729,3.4347)$ & $(3.2386,3.2787,3.4375)$ & $(3.2390,3.2785,3.4441)$ & $(3.2391,3.2774,3.4553)$ & $(3.2420,3.2873,3.4897)$ & $(3.2468,3.3333,3.6479)$ \\
\hline & $(0.2360,0.2333,0.2286)$ & $(0.2365,0.2353,0.2288)$ & $(0.2365,0.2346,0.2286)$ & $(0.2363,0.2331,0.2283)$ & $(0.2365,0.2333,0.2270)$ & $(0.2343,0.2343,0.2185)$ \\
\hline \multirow[t]{2}{*}{2.0} & $(3.7267,3.7635,3.9063)$ & $(3.7270,3.7639,3.9062)$ & $(3.7270,3.7660,3.9099)$ & $(3.7280,3.7688,3.9167)$ & $(3.7298,3.7716,3.9489)$ & $(3.7393,3.8047,4.1040)$ \\
\hline & $(0.4917,0.4855,0.4560)$ & $(0.4918,0.4854,0.4543)$ & $(0.4914,0.4855,0.4519)$ & $(0.4917,0.4854,0.4481)$ & $(0.4912,0.4805,0.4426)$ & $(0.4899,0.4716,0.4172)$ \\
\hline
\end{tabular}

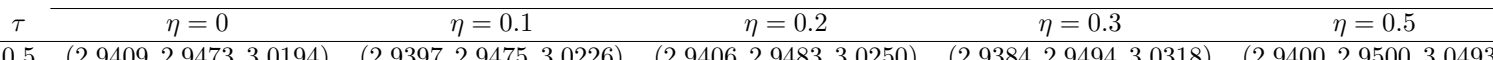
$\begin{array}{llllll}(0.1625,0.1542,0.1348) & (0.1621,0.1542,0.1352) & (0.1623,0.1540,0.1342) & (0.1613,0.1537,0.1334) & (0.1613,0.1517,0.1296) \\ (2.7899,2.7924,2.8496) & (2.7904,2.7945,2.8498) & (2.7868,2.7937,2.8549) & (2.7876,2.7945,2.8593) & (2.7876,2.7973,2.8778)\end{array}$ $\begin{array}{lllllll}(0.1134,0.1074,0.0920) & (0.1135,0.1079,0.0916) & (0.1124,0.1074,0.0915) & (0.1125,0.1071,0.0904) & (0.1121,0.1064,0.0877)\end{array}$ $8 \quad(2.5568,2.5524,2.5778) \quad(2.5601,2.5510,2.5796)$ $(0.0596,0.0567,0.0466) \quad(0.0602,0.0565,0.0466)$

$1.3 \quad(2.9306,2.9471,3.0441) \quad(2.9289,2.9475,3.0452)$ $(0.1589,0.1542,0.1420) \quad(0.1583,0.1542,0.1418)$

$1.5 \quad(3.2976,3.3260,3.4417) \quad(3.2960,3.3269,3.4455)$ $(0.3195,0.3156,0.3010) \quad(0.3186,0.3159,0.3020)$

$2.0 \quad(3.6953,3.7285,3.8487) \quad(3.6954,3.7266,3.8499)$ $(0.6418,0.6359,0.5966) \quad(0.6418,0.6330,0.5958)$

$(2.5547,2.5525,2.5821) \quad(2.5608,2.5528,2.5880)$ $(0.0593,0.0566,0.0462) \quad(0.0603,0.0565,0.0458)$ $(2.9323,2.9486,3.0518) \quad(2.9307,2.9500,3.0598)$ $(0.1594,0.1541,0.1420) \quad(0.1586,0.1539,0.1416)$ $(3.2946,3.3261,3.4475) \quad(3.2969,3.3303,3.4543)$ $(0.3176,0.3146,0.3000) \quad(0.3186,0.3156,0.2985)$ $(3.6974,3.7273,3.8564) \quad(3.6960,3.7298,3.8633)$ $(0.6438,0.6320,0.5959)$
$(2.5570,2.5491,2.6105)$ $(0.0595,0.0554,0.0446)$ $(2.9344,2.9538,3.0851)$ $(0.1594,0.1529,0.1398)$ $(3.3006,3.3343,3.4758$ $(0.3195,0.3137,0.2938)$ $(3.6980,3.7360,3.8926)$ $(0.6413,0.6296,0.5877)$ $\eta=1$ $(2.9426,2.9607,3.1553)$ $(0.1596,0.1448,0.1174)$ $(2.7899,2.8078,2.9896)$ $(0.1112,0.1021,0.0781)$ $(2.5572,2.5502,2.7473)$ $(0.0590,0.0527,0.0393)$ $(2.9361,2.9715,3.2329)$ $(0.1574,0.1481,0.1397)$ $(3.3076,3.3598,3.5987)$ $(0.3186,0.3082,0.2825)$ $(3.7071,3.7672,4.0163)$ $(0.6408,0.6212,0.5526)$ $=15$

\begin{tabular}{|c|c|c|c|c|c|c|}
\hline \multirow[b]{2}{*}{$\tau$} & \\
\hline & $\eta=0$ & $\eta=0.1$ & $\eta=0.2$ & $\eta=0.3$ & $\eta=0.5$ & $\eta=1$ \\
\hline \multirow[t]{2}{*}{0.5} & $(3.0561,3.0710,3.1077)$ & $(3.0579,3.0732,3.1065)$ & $(3.0582,3.0751,3.1071)$ & $(3.0560,3.0732,3.1117)$ & $(3.0578,3.0806,3.1220)$ & $(3.0601,3.0926,3.2109)$ \\
\hline & $(0.2448,0.2390,0.2043)$ & $(0.2458,0.2399,0.2031)$ & $(0.2458,0.2404,0.2014)$ & $(0.2443,0.2385,0.2001)$ & $(0.2446,0.2395,0.1943)$ & $(0.2424,0.2319,0.1845)$ \\
\hline \multirow[t]{2}{*}{0.7} & $(2.8987,2.9072,2.9467)$ & $(2.8993,2.9049,2.9484)$ & $(2.8976,2.9063,2.9511)$ & $(2.8978,2.9068,2.9544)$ & $(2.8987,2.9090,2.9659)$ & $(2.9017,2.9190,3.0384)$ \\
\hline & $(0.1650,0.1599,0.1392)$ & $(0.1652,0.1588,0.1394)$ & $(0.1644,0.1590,0.1389)$ & $(0.1643,0.1586,0.1376)$ & $(0.1643,0.1577,0.1339)$ & $(0.1634,0.1528,0.1213)$ \\
\hline \multirow[t]{2}{*}{0.8} & $(2.6805,2.6778,2.6949)$ & $(2.6777,2.6784,2.6958)$ & $(2.6797,2.6790,2.6948)$ & $(2.6799,2.6779,2.6995)$ & $(2.6786,2.6768,2.7129)$ & $(2.6791,2.6776,2.7853)$ \\
\hline & $(0.0869,0.0831,0.0711)$ & $(0.0861,0.0832,0.0710)$ & $(0.0866,0.0832,0.0701)$ & $(0.0866,0.0827,0.0698)$ & $(0.0861,0.0817,0.0683)$ & $(0.0855,0.0784,0.0603)$ \\
\hline \multirow[t]{2}{*}{1.3} & $(2.9936,3.0094,3.0806)$ & $(2.9956,3.0095,3.0787)$ & $(2.9939,3.0097,3.0808)$ & $(2.9962,3.0119,3.0869)$ & $(2.9945,3.0123,3.1045)$ & $(2.9970,3.0285,3.2039)$ \\
\hline & $(0.2110,0.2070,0.1921)$ & $(0.2120,0.2069,0.1906)$ & $(0.2110,0.2065,0.1896)$ & $(0.2120,0.2068,0.1892)$ & $(0.2105,0.2044,0.1867)$ & $(0.2088,0.2002,0.1816)$ \\
\hline \multirow[t]{2}{*}{1.5} & $(3.3332,3.3560,3.4441)$ & $(3.3311,3.3553,3.4423)$ & $(3.3347,3.3559,3.4451)$ & $(3.3341,3.3561,3.4519)$ & $(3.3356,3.3617,3.4735)$ & $(3.3395,3.3838,3.5575)$ \\
\hline & $(0.4396,0.4343,0.4111)$ & $(0.4377,0.4333,0.4084)$ & $(0.4406,0.4328,0.4069)$ & $(0.4396,0.4313,0.4062)$ & $(0.4396,0.4309,0.4038)$ & $(0.4367,0.4250,0.3800)$ \\
\hline \multirow[t]{2}{*}{2.0} & $(3.6038,3.6315,3.7431)$ & $(3.6035,3.6319,3.7422)$ & $(3.6059,3.6329,3.7479)$ & $(3.6043,3.6350,3.7546)$ & $(3.6065,3.6411,3.7774)$ & $(3.6139,3.6684,3.8887)$ \\
\hline & $(0.8186,0.8112,0.7824)$ & $(0.8176,0.8111,0.7766)$ & $(0.8234,0.8105,0.7781)$ & $(0.8176,0.8107,0.7746)$ & $(0.8190,0.8098,0.7664)$ & $(0.8186,0.8010,0.7377)$ \\
\hline
\end{tabular}

Table 6: Optimal values $\lambda^{*}$ (first row of each block) and $K^{*}$ (second row of each block) of two-sided EWMA- $\gamma^{2}$ control charts in the presence of measurement errors for $\gamma_{0}=0.05$ (left side), $\gamma_{0}=0.1$ (middle) and $\gamma_{0}=0.2$ (right side), for different values of $\eta, \theta=0.05$, $\tau, n, B=1, m=1$. 


\begin{tabular}{|c|c|c|c|c|c|c|}
\hline \multirow[b]{2}{*}{$\tau$} & \multicolumn{6}{|c|}{$n=5$} \\
\hline & $\eta=0$ & $\eta=0.1$ & $\eta=0.2$ & $\eta=0.3$ & $\eta=0.5$ & $\eta=1$ \\
\hline 0.5 & $(9.50,9.95,12.32)$ & $(9.50,9.96,12.36)$ & $(9.51,9.98,12.47)$ & $(9.51,10.01,12.67)$ & $(9.54,10.12,13.32)$ & $(9.65,10.64,17.04)$ \\
\hline 0.7 & $(14.07,14.75,18.63)$ & $(14.08,14.76,18.69)$ & $(14.08,14.79,18.89)$ & $(14.09,14.84,19.24)$ & $(14.13,15.00,20.42)$ & $(14.29,15.81,27.51)$ \\
\hline 0.8 & $(28.07,29.55,39.49)$ & $(28.07,29.57,39.69)$ & $(28.08,29.63,40.28)$ & $(28.11,29.74,41.30)$ & $(28.18,30.11,44.89)$ & $(28.53,32.04,69.81)$ \\
\hline 1.3 & $(15.06,15.21,16.08)$ & $(15.06,15.21,16.09)$ & $(15.06,15.22,16.13)$ & $(15.06,15.23,16.20)$ & $(15.07,15.27,16.41)$ & $(15.11,15.46,17.44)$ \\
\hline 1.5 & $(5.93,6.02,6.38)$ & $(5.93,6.02,6.39)$ & $(5.93,6.03,6.40)$ & $(5.94,6.03,6.43)$ & $(5.94,6.05,6.51)$ & $(5.96,6.14,6.90)$ \\
\hline \multirow[t]{2}{*}{2.0} & $(2.55,2.59,2.75)$ & $(2.55,2.59,2.75)$ & $(2.56,2.59,2.76)$ & $(2.56,2.60,2.77)$ & $(2.56,2.61,2.80)$ & $(2.57,2.64,2.97)$ \\
\hline & \multicolumn{6}{|c|}{$n=7$} \\
\hline$\tau$ & $\eta=0$ & $\eta=0.1$ & $\eta=0.2$ & $\eta=0.3$ & $\eta=0.5$ & $\eta=1$ \\
\hline 0.5 & $(6.99,7.27,8.60)$ & $(6.99,7.27,8.62)$ & $(7.00,7.28,8.68)$ & $(7.00,7.30,8.78)$ & $(7.01,7.36,9.13)$ & $(7.08,7.67,10.98)$ \\
\hline 0.7 & $(10.39,10.81,12.96)$ & $(10.39,10.81,12.99)$ & $(10.39,10.83,13.10)$ & $(10.40,10.86,13.27)$ & $(10.42,10.95,13.87)$ & $(10.52,11.43,17.26)$ \\
\hline 0.8 & $(20.99,21.90,27.22)$ & $(20.99,21.91,27.32)$ & $(21.00,21.95,27.61)$ & $(21.02,22.02,28.10)$ & $(21.06,22.23,29.80)$ & $(21.28,23.34,40.69)$ \\
\hline 1.3 & $(11.40,11.52,12.16)$ & $(11.40,11.52,12.17)$ & $(11.40,11.53,12.20)$ & $(11.40,11.54,12.25)$ & $(11.41,11.57,12.40)$ & $(11.44,11.71,13.16)$ \\
\hline 1.5 & $(4.45,4.51,4.79)$ & $(4.45,4.51,4.79)$ & $(4.45,4.52,4.80)$ & $(4.45,4.52,4.82)$ & $(4.45,4.53,4.88)$ & $(4.47,4.60,5.18)$ \\
\hline \multirow[t]{2}{*}{2.0} & $(1.95,1.98,2.10)$ & $(1.95,1.98,2.11)$ & $(1.95,1.98,2.11)$ & $(1.95,1.98,2.12)$ & $(1.95,1.99,2.15)$ & $(1.96,2.02,2.28)$ \\
\hline & \multicolumn{6}{|c|}{$n=10$} \\
\hline$\tau$ & $\eta=0$ & $\eta=0.1$ & $\eta=0.2$ & $\eta=0.3$ & $\eta=0.5$ & $\eta=1$ \\
\hline 0.5 & $(5.13,5.30,6.04)$ & $(5.13,5.30,6.05)$ & $(5.13,5.31,6.09)$ & $(5.14,5.32,6.15)$ & $(5.14,5.35,6.33)$ & $(5.19,5.52,7.29)$ \\
\hline 0.7 & $(7.63,7.88,9.09)$ & $(7.63,7.88,9.10)$ & $(7.63,7.89,9.16)$ & $(7.63,7.91,9.25)$ & $(7.65,7.97,9.56)$ & $(7.71,8.24,11.25)$ \\
\hline 0.8 & $(15.58,16.13,19.02)$ & $(15.58,16.13,19.07)$ & $(15.58,16.16,19.21)$ & $(15.59,16.20,19.46)$ & $(15.62,16.32,20.29)$ & $(15.75,16.96,25.21)$ \\
\hline 1.3 & $(8.57,8.67,9.14)$ & $(8.57,8.67,9.14)$ & $(8.57,8.67,9.16)$ & $(8.57,8.68,9.20)$ & $(8.58,8.70,9.31)$ & $(8.60,8.81,9.85)$ \\
\hline 1.5 & $(3.33,3.38,3.58)$ & $(3.33,3.38,3.59)$ & $(3.33,3.38,3.60)$ & $(3.33,3.39,3.61)$ & $(3.33,3.40,3.65)$ & $(3.35,3.45,3.87)$ \\
\hline \multirow[t]{2}{*}{2.0} & $(1.52,1.54,1.63)$ & $(1.52,1.54,1.63)$ & $(1.52,1.54,1.64)$ & $(1.52,1.54,1.64)$ & $(1.52,1.55,1.66)$ & $(1.53,1.57,1.76)$ \\
\hline & \multicolumn{6}{|c|}{$n=15$} \\
\hline$\tau$ & $\eta=0$ & $\eta=0.1$ & $\eta=0.2$ & $\eta=0.3$ & $\eta=0.5$ & $\eta=1$ \\
\hline 0.5 & $(3.62,3.74,4.17)$ & $(3.62,3.74,4.17)$ & $(3.62,3.74,4.19)$ & $(3.62,3.75,4.21)$ & $(3.63,3.77,4.30)$ & $(3.66,3.89,4.79)$ \\
\hline 0.7 & $(5.42,5.56,6.23)$ & $(5.42,5.57,6.24)$ & $(5.42,5.57,6.26)$ & $(5.42,5.58,6.31)$ & $(5.43,5.61,6.47)$ & $(5.47,5.77,7.30)$ \\
\hline 0.8 & $(11.17,11.49,13.02)$ & $(11.17,11.49,13.04)$ & $(11.17,11.51,13.12)$ & $(11.18,11.53,13.24)$ & $(11.19,11.60,13.64)$ & $(11.27,11.95,15.85)$ \\
\hline 1.3 & $(6.25,6.32,6.65)$ & $(6.25,6.32,6.66)$ & $(6.25,6.33,6.67)$ & $(6.25,6.33,6.70)$ & $(6.25,6.35,6.77)$ & $(6.27,6.43,7.14)$ \\
\hline 1.5 & $(2.44,2.47,2.62)$ & $(2.44,2.47,2.62)$ & $(2.44,2.47,2.63)$ & $(2.44,2.48,2.64)$ & $(2.44,2.48,2.67)$ & $(2.45,2.52,2.82)$ \\
\hline 2.0 & $(1.21,1.23,1.28)$ & $(1.21,1.23,1.28)$ & $(1.21,1.23,1.28)$ & $(1.21,1.23,1.29)$ & $(1.21,1.23,1.30)$ & $(1.22,1.24,1.36)$ \\
\hline
\end{tabular}

Table 7: $A R L$ values of two-sided EWMA- $\gamma^{2}$ control charts in the presence of measurement errors for $\gamma_{0}=0.05$ (left side), $\gamma_{0}=0.1$ (middle) and $\gamma_{0}=0.2$ (right side), for different values of $\eta, \theta=0.05, \tau, n, B=1, m=1$. 


\begin{tabular}{|c|c|c|c|c|c|c|}
\hline \multirow[b]{2}{*}{$\tau$} & \multicolumn{6}{|c|}{$n=5$} \\
\hline & $\theta=0$ & $\theta=0.01$ & $\theta=0.02$ & $\theta=0.03$ & $\theta=0.04$ & $\theta=0.05$ \\
\hline 0.5 & $(9.30,9.84,12.73)$ & $(9.34,9.87,12.71)$ & $(9.39,9.90,12.68)$ & $(9.43,9.94,12.66)$ & $(9.47,9.97,12.64)$ & $(9.51,10.00,12.62)$ \\
\hline 0.7 & $(13.60,14.40,19.17)$ & $(13.70,14.48,19.16)$ & $(13.80,14.57,19.15)$ & $(13.90,14.65,19.15)$ & $(13.99,14.74,19.15)$ & $(14.09,14.83,19.16)$ \\
\hline 0.8 & $(26.81,28.52,41.04)$ & $(27.06,28.75,41.03)$ & $(27.32,28.99,41.02)$ & $(27.58,29.23,41.02)$ & $(27.84,29.47,41.04)$ & $(28.10,29.72,41.06)$ \\
\hline 1.3 & $(13.85,14.04,15.05)$ & $(14.09,14.27,15.27)$ & $(14.33,14.51,15.50)$ & $(14.57,14.75,15.73)$ & $(14.82,14.99,15.95)$ & $(15.06,15.23,16.18)$ \\
\hline 1.5 & $(5.39,5.49,5.88)$ & $(5.50,5.60,5.99)$ & $(5.61,5.70,6.10)$ & $(5.72,5.81,6.20)$ & $(5.83,5.92,6.31)$ & $(5.94,6.03,6.42)$ \\
\hline \multirow[t]{2}{*}{2.0} & $(2.30,2.34,2.51)$ & $(2.35,2.39,2.56)$ & $(2.40,2.44,2.61)$ & $(2.45,2.49,2.66)$ & $(2.50,2.54,2.71)$ & $(2.56,2.60,2.77)$ \\
\hline & \multicolumn{6}{|c|}{$n=7$} \\
\hline$\tau$ & $\theta=0$ & $\theta=0.01$ & $\theta=0.02$ & $\theta=0.03$ & $\theta=0.04$ & $\theta=0.05$ \\
\hline 0.5 & $(6.84,7.16,8.77)$ & $(6.87,7.19,8.76)$ & $(6.90,7.22,8.76)$ & $(6.94,7.24,8.76)$ & $(6.97,7.27,8.76)$ & $(7.00,7.30,8.76)$ \\
\hline 0.7 & $(10.03,10.52,13.11)$ & $(10.11,10.58,13.13)$ & $(10.18,10.65,13.15)$ & $(10.25,10.72,13.18)$ & $(10.33,10.78,13.20)$ & $(10.40,10.85,13.23)$ \\
\hline 0.8 & $(20.01,21.06,27.55)$ & $(20.21,21.24,27.63)$ & $(20.41,21.43,27.71)$ & $(20.61,21.62,27.80)$ & $(20.81,21.81,27.89)$ & $(21.01,22.00,27.98)$ \\
\hline 1.3 & $(10.47,10.62,11.36)$ & $(10.65,10.80,11.53)$ & $(10.84,10.98,11.70)$ & $(11.02,11.16,11.88)$ & $(11.21,11.35,12.06)$ & $(11.40,11.54,12.23)$ \\
\hline 1.5 & $(4.04,4.11,4.41)$ & $(4.12,4.19,4.49)$ & $(4.20,4.27,4.57)$ & $(4.28,4.36,4.65)$ & $(4.36,4.44,4.73)$ & $(4.45,4.52,4.82)$ \\
\hline \multirow[t]{2}{*}{2.0} & $(1.77,1.80,1.94)$ & $(1.81,1.84,1.97)$ & $(1.84,1.87,2.01)$ & $(1.88,1.91,2.04)$ & $(1.91,1.95,2.08)$ & $(1.95,1.98,2.12)$ \\
\hline & \multicolumn{6}{|c|}{$n=10$} \\
\hline$\tau$ & $\theta=0$ & $\theta=0.01$ & $\theta=0.02$ & $\theta=0.03$ & $\theta=0.04$ & $\theta=0.05$ \\
\hline 0.5 & $(5.02,5.22,6.11)$ & $(5.04,5.24,6.11)$ & $(5.07,5.25,6.11)$ & $(5.09,5.27,6.12)$ & $(5.11,5.29,6.13)$ & $(5.13,5.31,6.13)$ \\
\hline 0.7 & $(7.36,7.65,9.08)$ & $(7.41,7.70,9.11)$ & $(7.47,7.75,9.14)$ & $(7.52,7.80,9.17)$ & $(7.58,7.85,9.20)$ & $(7.63,7.91,9.23)$ \\
\hline 0.8 & $(14.83,15.46,18.90)$ & $(14.98,15.60,19.00)$ & $(15.13,15.75,19.09)$ & $(15.28,15.89,19.19)$ & $(15.44,16.04,19.29)$ & $(15.59,16.19,19.40)$ \\
\hline 1.3 & $(7.86,7.98,8.51)$ & $(8.00,8.12,8.65)$ & $(8.14,8.26,8.78)$ & $(8.28,8.40,8.92)$ & $(8.43,8.54,9.05)$ & $(8.57,8.68,9.19)$ \\
\hline 1.5 & $(3.03,3.08,3.31)$ & $(3.09,3.14,3.37)$ & $(3.15,3.20,3.43)$ & $(3.21,3.26,3.49)$ & $(3.27,3.32,3.55)$ & $(3.33,3.38,3.61)$ \\
\hline \multirow[t]{2}{*}{2.0} & $(1.40,1.42,1.52)$ & $(1.42,1.45,1.54)$ & $(1.45,1.47,1.57)$ & $(1.47,1.49,1.59)$ & $(1.49,1.52,1.62)$ & $(1.52,1.54,1.64)$ \\
\hline & \multicolumn{6}{|c|}{$n=15$} \\
\hline$\tau$ & $\theta=0$ & $\theta=0.01$ & $\theta=0.02$ & $\theta=0.03$ & $\theta=0.04$ & $\theta=0.05$ \\
\hline 0.5 & $(3.52,3.66,4.17)$ & $(3.54,3.68,4.18)$ & $(3.56,3.70,4.19)$ & $(3.58,3.71,4.19)$ & $(3.60,3.73,4.20)$ & $(3.62,3.75,4.21)$ \\
\hline 0.7 & $(5.22,5.39,6.17)$ & $(5.26,5.43,6.19)$ & $(5.30,5.47,6.22)$ & $(5.34,5.50,6.25)$ & $(5.38,5.54,6.27)$ & $(5.42,5.58,6.30)$ \\
\hline 0.8 & $(10.62,10.98,12.77)$ & $(10.73,11.09,12.86)$ & $(10.84,11.20,12.94)$ & $(10.95,11.30,13.03)$ & $(11.06,11.41,13.12)$ & $(11.18,11.52,13.21)$ \\
\hline 1.3 & $(5.73,5.82,6.19)$ & $(5.83,5.92,6.29)$ & $(5.94,6.02,6.39)$ & $(6.04,6.12,6.49)$ & $(6.14,6.23,6.59)$ & $(6.25,6.33,6.69)$ \\
\hline 1.5 & $(2.22,2.26,2.42)$ & $(2.27,2.30,2.46)$ & $(2.31,2.35,2.51)$ & $(2.35,2.39,2.55)$ & $(2.39,2.43,2.59)$ & $(2.44,2.48,2.63)$ \\
\hline 2.0 & $(1.15,1.16,1.22)$ & $(1.16,1.17,1.23)$ & $(1.17,1.19,1.24)$ & $(1.19,1.20,1.26)$ & $(1.20,1.21,1.27)$ & $(1.21,1.23,1.29)$ \\
\hline
\end{tabular}

Table 8: $A R L$ values of two-sided EWMA- $\gamma^{2}$ control charts in the presence of measurement errors for $\gamma_{0}=0.05$ (left side), $\gamma_{0}=0.1$ (middle) and $\gamma_{0}=0.2$ (right side), for different values of $\theta, \eta=0.28, \tau, n, B=1, m=1$. 


\begin{tabular}{|c|c|c|c|c|c|}
\hline \multirow[b]{2}{*}{$\tau$} & \multicolumn{5}{|c|}{$n=5$} \\
\hline & $B=1$ & $B=2$ & $B=3$ & $B=4$ & $B=5$ \\
\hline 0.5 & $(9.51,10.00,12.62)$ & $(9.40,9.88,12.43)$ & $(9.36,9.84,12.40)$ & $(9.34,9.83,12.39)$ & $(9.33,9.82,12.39)$ \\
\hline 0.7 & $(14.09,14.83,19.16)$ & $(13.83,14.55,18.73)$ & $(13.75,14.47,18.65)$ & $(13.71,14.43,18.62)$ & $(13.68,14.40,18.60)$ \\
\hline 0.8 & $(28.10,29.72,41.06)$ & $(27.43,28.98,39.77)$ & $(27.20,28.75,39.52)$ & $(27.10,28.64,39.43)$ & $(27.03,28.58,39.38)$ \\
\hline 1.3 & $(15.06,15.23,16.18)$ & $(14.45,14.61,15.53)$ & $(14.25,14.41,15.33)$ & $(14.15,14.31,15.23)$ & $(14.09,14.25,15.17)$ \\
\hline 1.5 & $(5.94,6.03,6.42)$ & $(5.66,5.75,6.12)$ & $(5.57,5.66,6.02)$ & $(5.53,5.61,5.98)$ & $(5.50,5.59,5.95)$ \\
\hline \multirow[t]{2}{*}{2.0} & $(2.56,2.60,2.77)$ & $(2.43,2.46,2.62)$ & $(2.38,2.42,2.58)$ & $(2.36,2.40,2.56)$ & $(2.35,2.39,2.54)$ \\
\hline & \multicolumn{5}{|c|}{$n=7$} \\
\hline$\tau$ & $B=1$ & $B=2$ & $B=3$ & $B=4$ & $B=5$ \\
\hline 0.5 & $(7.00,7.30,8.76)$ & $(6.91,7.21,8.63)$ & $(6.89,7.18,8.61)$ & $(6.87,7.17,8.60)$ & $(6.87,7.16,8.60)$ \\
\hline 0.7 & $(10.40,10.85,13.23)$ & $(10.21,10.65,12.95)$ & $(10.14,10.58,12.88)$ & $(10.11,10.55,12.86)$ & $(10.10,10.54,12.85)$ \\
\hline 0.8 & $(21.01,22.00,27.98)$ & $(20.49,21.45,27.15)$ & $(20.33,21.27,26.96)$ & $(20.24,21.19,26.89)$ & $(20.19,21.14,26.84)$ \\
\hline 1.3 & $(11.40,11.54,12.23)$ & $(10.93,11.06,1$ & $(10.77,10.91,11$ & $(10.69,10.83,1$ & $(10.65,10.78,11.46)$ \\
\hline 1.5 & $(4.45,4.52$ & $(4.24,4.3$ & $(4.17,4.2$ & $(4.14,4.2$ & $(4.12,4.1$ \\
\hline \multirow[t]{2}{*}{2.0} & $(1.95,1.98,2.12)$ & $(1.86,1.89,2.02)$ & $(1.83,1.86,1.98)$ & $(1.81,1.84,1.97)$ & $(1.81,1.84,1.96)$ \\
\hline & \multicolumn{5}{|c|}{$n=10$} \\
\hline$\tau$ & $B=1$ & $B=2$ & $B=3$ & $B=4$ & $B=5$ \\
\hline 0.5 & $(5.13,5.31,6.13)$ & $(5.07,5.25,6.05)$ & $(5.05,5.23,6.03)$ & $(5.04,5.22,6.02)$ & $(5.04,5.22,6.02)$ \\
\hline 0.7 & $(7.63,7.91,9$ & $(7.49,7.7$ & $(7.44,7.71,8$ & $(7.42,7.69,8$ & $(7.41,7.67,8.96)$ \\
\hline 0.8 & $(15.59,16.19,19.40)$ & $(15.20,15.77,18.85)$ & $(15.07,15.64,18.70)$ & $(15.00,15.58,18.64)$ & $(14.97,15.54,18.61)$ \\
\hline 1.3 & $(8.57,8.68,9.19)$ & $(8.21,8.32,8.81)$ & $(8.09,8.20,8.69)$ & $(8.03,8.14,8.63)$ & $(8.00,8.11,8.59)$ \\
\hline 1.5 & $(3.33,3.38,3.61)$ & $(3.18,3.23,3.44)$ & $(3.13,3.18,3.39)$ & $(3.10,3.15,3.36)$ & $(3.09,3.14,3.35)$ \\
\hline \multirow[t]{2}{*}{2.0} & $(1.52,1.54,1.64)$ & $(1.46,1.48,1.57)$ & $(1.44,1.46,1.55)$ & $(1.43,1.45,1.54)$ & $(1.42,1.44,1.53)$ \\
\hline & \multicolumn{5}{|c|}{$n=15$} \\
\hline$\tau$ & $B=1$ & $B=2$ & $B=3$ & $B=4$ & $B=5$ \\
\hline 0.5 & $(3.62,3.75,4.21)$ & $(3.57,3.70,4.16)$ & $(3.55,3.68,4.15)$ & $(3.55,3.67,4.14)$ & $(3.54,3.67,4.14)$ \\
\hline 0.7 & $(5.42,5.58,6.30)$ & $(5.32,5.47,6.17)$ & $(5.29,5.44,6.14)$ & $(5.27,5.42,6.12)$ & $(5.26,5.41,6.12)$ \\
\hline 0.8 & $(11.18,11.52,13.21)$ & $(10.89,11.22,12.84)$ & $(10.80,11.13,12.74)$ & $(10.75,11.08,12.69)$ & $(10.72,11.05,12.67)$ \\
\hline 1.3 & $(6.25,6.33,6.69)$ & $(5.99,6.07,6.41)$ & $(5.90,5.98,6.32)$ & $(5.86,5.94,6.28)$ & $(5.83,5.91,6.25)$ \\
\hline 1.5 & $(2.44,2.48,2.63)$ & $(2.33,2.37,2.52)$ & $(2.29,2.33,2.48)$ & $(2.27,2.31,2.46)$ & $(2.26,2.30,2.45)$ \\
\hline 2.0 & $(1.21,1.23,1.29)$ & $(1.18,1.19,1.25)$ & $(1.17,1.18,1.23)$ & $(1.16,1.18,1.23)$ & $(1.16,1.17,1.22)$ \\
\hline
\end{tabular}

Table 9: $A R L$ values of two-sided EWMA- $\gamma^{2}$ control charts in the presence of measurement errors for $\gamma_{0}=0.05$ (left side), $\gamma_{0}=0.1$ (middle) and $\gamma_{0}=0.2$ (right side), for different values of $B, \tau, n, \eta=0.28, \theta=0.01, m=1$. 


\begin{tabular}{|c|c|c|c|c|c|}
\hline \multirow[b]{2}{*}{$\tau$} & \multicolumn{5}{|c|}{$n=5$} \\
\hline & $m=1$ & $m=3$ & $m=5$ & $m=7$ & $m=10$ \\
\hline 0.5 & $(9.51,10.00,12.62)$ & $(9.51,9.97,12.42)$ & $(9.50,9.96,12.38)$ & $(9.50,9.96,12.36)$ & $(9.50,9.96,12.35)$ \\
\hline 0.7 & $(14.09,14.83,19.16)$ & $(14.08,14.78,18.80)$ & $(14.08,14.77,18.73)$ & $(14.08,14.76,18.70)$ & $(14.08,14.76,18.68)$ \\
\hline 0.8 & $(28.10,29.72,41.06)$ & $(28.08,29.60,40.00)$ & $(28.07,29.58,39.80)$ & $(28.07,29.57,39.71)$ & $(28.07,29.56,39.64)$ \\
\hline 1.3 & $(15.06,15.23,16.18)$ & $(15.06,15.21,16.11)$ & $(15.06,15.21,16.10)$ & $(15.06,15.21,16.09)$ & $(15.06,15.21,16.09)$ \\
\hline 1.5 & $(5.94,6.03,6.42)$ & $(5.93,6.02,6.40)$ & $(5.93,6.02,6.39)$ & $(5.93,6.02,6.39)$ & $(5.93,6.02,6.39)$ \\
\hline \multirow[t]{2}{*}{2.0} & $(2.56,2.60,2.77)$ & $(2.56,2.59,2.75)$ & $(2.56,2.59,2.75)$ & $(2.56,2.59,2.75)$ & $(2.55,2.59,2.75)$ \\
\hline & \multicolumn{5}{|c|}{$n=7$} \\
\hline$\tau$ & $m=1$ & $m=3$ & $m=5$ & $m=7$ & $m=10$ \\
\hline 0.5 & $(7.00,7.30,8.76)$ & $(6.99,7.28,8.65)$ & $(6.99,7.27,8.63)$ & $(6.99,7.27,8.62)$ & $(6.99,7.27,8.61)$ \\
\hline 0.7 & $(10.40,10.85,13.23)$ & $(10.39,10.82,13.05)$ & $(10.39,10.81,13.01)$ & $(10.39,10.81,12.99)$ & $(10.39,10.81,12.98)$ \\
\hline 0.8 & $(21.01,22.00,27.98)$ & $(21.00,21.93,27.47)$ & $(20.99,21.92,27.37)$ & $(20.99,21.91,27.33)$ & $(20.99,21.91,27.30)$ \\
\hline 1.3 & $(11.40,11.54,12.23)$ & $(11.40,11.53,1$ & $(11.40,11.52,12$. & $(11.40,11.5$ & $(11.40,11.52,12.17)$ \\
\hline 1.5 & $(4.45,4.52,4$ & $(4.45,4$ & $(4.45,4.51$ & $(4.45,4$ & $(4.45,4.51,4$ \\
\hline \multirow[t]{2}{*}{2.0} & $(1.95,1.98,2.12)$ & $(1.95,1.98,2.11)$ & $(1.95,1.98,2.11)$ & $(1.95,1.98,2.11)$ & $(1.95,1.98,2.11)$ \\
\hline & \multicolumn{5}{|c|}{$n=10$} \\
\hline$\tau$ & $m=1$ & $m=3$ & $m=5$ & $m=7$ & $m=10$ \\
\hline 0.5 & $(5.13,5.31,6.13)$ & $(5.13,5.30,6.07)$ & $(5.13,5.30,6.06)$ & $(5.13,5.30,6.06)$ & $(5.13,5.30,6.05)$ \\
\hline 0.7 & $(7.63,7.91,9.23)$ & $(7.63,7$. & $(7.63,7.88,9.11)$ & $(7.63,7.8$ & $(7.63,7.88,9.10)$ \\
\hline 0.8 & $(15.59,16.19,19.40)$ & $(15.58,16.15,19.14)$ & $(15.58,16.14,19.09)$ & $(15.58,16.13,19.07)$ & $(15.58,16.13,19.06)$ \\
\hline 1.3 & $(8.57,8.68,9.19)$ & $(8.57,8.67,9.15)$ & $(8.57,8.67,9.15)$ & $(8.57,8.67,9.14)$ & $(8.57,8.67,9.14)$ \\
\hline 1.5 & $(3.33,3.38,3.61)$ & $(3.33,3.38,3.59)$ & $(3.33,3.38,3.59)$ & $(3.33,3.38,3.59)$ & $(3.33,3.38,3.59)$ \\
\hline \multirow[t]{2}{*}{2.0} & $(1.52,1.54,1.64)$ & $(1.52,1.54,1.64)$ & $(1.52,1.54,1.63)$ & $(1.52,1.54,1.63)$ & $(1.52,1.54,1.63)$ \\
\hline & \multicolumn{5}{|c|}{$n=15$} \\
\hline$\tau$ & $m=1$ & $m=3$ & $m=5$ & $m=7$ & $m=10$ \\
\hline 0.5 & $(3.62,3.75,4.21)$ & $(3.62,3.74,4.18)$ & $(3.62,3.74,4.18)$ & $(3.62,3.74,4.17)$ & $(3.62,3.74,4.17)$ \\
\hline 0.7 & $(5.42,5.58,6.30)$ & $(5.42,5.57,6.25)$ & $(5.42,5.57,6.24)$ & $(5.42,5.57,6.24)$ & $(5.42,5.57,6.23)$ \\
\hline 0.8 & $(11.18,11.52,13.21)$ & $(11.17,11.50,13.08)$ & $(11.17,11.49,13.06)$ & $(11.17,11.49,13.05)$ & $(11.17,11.49,13.04)$ \\
\hline 1.3 & $(6.25,6.33,6.69)$ & $(6.25,6.33,6.67)$ & $(6.25,6.33,6.66)$ & $(6.25,6.32,6.66)$ & $(6.25,6.32,6.66)$ \\
\hline 1.5 & $(2.44,2.48,2.63)$ & $(2.44,2.47,2.62)$ & $(2.44,2.47,2.62)$ & $(2.44,2.47,2.62)$ & $(2.44,2.47,2.62)$ \\
\hline 2.0 & $(1.21,1.23,1.29)$ & $(1.21,1.23,1.28)$ & $(1.21,1.23,1.28)$ & $(1.21,1.23,1.28)$ & $(1.21,1.23,1.28)$ \\
\hline
\end{tabular}

Table 10: $A R L$ values of two-sided EWMA- $\gamma^{2}$ control charts in the presence of measurement errors for $\gamma_{0}=0.05$ (left side), $\gamma_{0}=0.1$ (middle) and $\gamma_{0}=0.2$ (right side), for different values of $m, \tau, n, \eta=0.28, \theta=0.05, B=1$. 


\begin{tabular}{ccccccc}
\hline$\eta$ & $\theta$ & $\gamma_{0}$ & $n=5$ & $n=7$ & $n=10$ & $n=15$ \\
\hline \hline 0.10 & 0.01 & 0.05 & $(0.0501,2.1425)$ & $(0.0502,2.1942)$ & $(0.0578,2.2619)$ & $(0.0690,2.3348)$ \\
& & & $(0.0501,2.6910)$ & $(0.0502,2.6473)$ & $(0.0579,2.6755)$ & $(0.0720,2.7294)$ \\
& & 0.10 & $(0.0500,2.0801)$ & $(0.0501,2.1401)$ & $(0.0552,2.2101)$ & $(0.0683,2.2989)$ \\
& & & $(0.0500,2.7439)$ & $(0.0509,2.7007)$ & $(0.0598,2.7286)$ & $(0.0725,2.7632)$ \\
& & 0.15 & $(0.0500,1.9813)$ & $(0.0500,2.0545)$ & $(0.0530,2.1307)$ & $(0.0651,2.2344)$ \\
& & $(0.0500,2.8353)$ & $(0.0531,2.7996)$ & $(0.0610,2.8025)$ & $(0.0732,2.8188)$ \\
& 0.20 & $(0.0500,1.8526)$ & $(0.0500,1.9425)$ & $(0.0502,2.0234)$ & $(0.0610,2.1454)$ \\
\hline 0.28 & 0.05 & 0.05 & $(0.0501,2.9688)$ & $(0.0556,2.9316)$ & $(0.0634,2.9100)$ & $(0.0740,2.8951)$ \\
& & & $(0.0501,2.6908)$ & $(0.0502,2.6471)$ & $(0.0574,2.6714)$ & $(0.0696,2.7150)$ \\
& & 0.10 & $(0.0500,2.0811)$ & $(0.0501,2.1409)$ & $(0.0540,2.2065)$ & $(0.0656,2.2924)$ \\
& & $(0.0500,2.7430)$ & $(0.0501,2.6929)$ & $(0.0588,2.7205)$ & $(0.0704,2.7505)$ \\
& 0.15 & $(0.0500,1.9833)$ & $(0.0500,2.0563)$ & $(0.0520,2.1279)$ & $(0.0627,2.2281)$ \\
& & $(0.0500,2.8333)$ & $(0.0519,2.7876)$ & $(0.0593,2.7887)$ & $(0.0710,2.8054)$ \\
& 0.20 & $(0.0500,1.8560)$ & $(0.0500,1.9455)$ & $(0.0500,2.0248)$ & $(0.0595,2.1419)$ \\
& & $(0.0501,2.9650)$ & $(0.0545,2.9199)$ & $(0.0615,2.8945)$ & $(0.0725,2.8848)$ \\
\hline
\end{tabular}

Table 11: Optimal couples $\left(\lambda^{*}, K^{*}\right)$ for downward EWMA- $\gamma^{2}$ (first row of each block) and optimal couples $\left(\lambda^{*}, K^{*}\right)$ for upward EWMA- $\gamma^{2}$ (second row of each block), in the presence of measurement errors, for different values of $\eta, \theta, n, \gamma_{0}, B=1$ and $m=1$.

\begin{tabular}{cccccccccccc}
\multicolumn{10}{c}{ Phase II } & \multicolumn{1}{c}{$\hat{X}^{*}$} \\
\hline$k$ & $X_{1}^{*}$ & $X_{2}^{*}$ & $X_{3}^{*}$ & $X_{4}^{*}$ & $X_{5}^{*}$ & $S_{i}^{*}$ & $X_{i}^{*}$ & $\hat{\gamma}^{*}$ & $\hat{\gamma}^{* 2}$ & $Z_{i}^{*}$ & $S_{i}^{*}$ \\
\hline 1 & 50.67 & 49.45 & 49.88 & 49.91 & 50.26 & 0.4571 & 50.0320 & 0.0091 & 0.00008 & 0.000108 & 0.000106 \\
2 & 49.59 & 50.21 & 50.23 & 49.58 & 49.76 & 0.3240 & 49.8757 & 0.0065 & 0.00004 & 0.000108 & 0.000102 \\
3 & 49.69 & 50.46 & 49.21 & 50.26 & 50.08 & 0.4969 & 49.9386 & 0.0100 & 0.00010 & 0.000108 & 0.000102 \\
4 & 49.48 & 50.71 & 50.04 & 49.99 & 50.54 & 0.4898 & 50.1502 & 0.0098 & 0.00010 & 0.000108 & 0.000102 \\
5 & 50.73 & 50.00 & 51.16 & 49.76 & 50.52 & 0.5624 & 50.4334 & 0.0112 & 0.00013 & 0.000109 & 0.000103 \\
6 & 49.42 & 51.25 & 50.28 & 51.85 & 49.45 & 1.0830 & 50.4510 & 0.0215 & 0.00046 & 0.000126 & 0.000126 \\
7 & 49.17 & 49.95 & 48.98 & 49.72 & 49.86 & 0.4307 & 49.5358 & 0.0087 & 0.00008 & 0.000124 & 0.000123 \\
8 & 50.09 & 50.43 & 50.02 & 49.77 & 49.41 & 0.3800 & 49.9448 & 0.0076 & 0.00006 & 0.000121 & 0.000119 \\
9 & 50.40 & 49.27 & 50.01 & 50.58 & 50.25 & 0.5100 & 50.1019 & 0.0102 & 0.00010 & 0.000120 & 0.000118 \\
10 & 49.11 & 50.28 & 51.22 & 49.79 & 51.21 & 0.9145 & 50.3215 & 0.0182 & 0.00033 & 0.000130 & 0.000131 \\
11 & 48.48 & 50.49 & 50.65 & 49.78 & 51.03 & 1.0047 & 50.0863 & 0.0201 & 0.00040 & 0.000144 & 0.000149 \\
12 & 48.01 & 49.34 & 50.71 & 48.00 & 49.21 & 1.1244 & 49.0523 & 0.0229 & 0.00052 & 0.000163 & 0.000173 \\
13 & 50.22 & 50.25 & 49.89 & 51.80 & 51.64 & 0.8886 & 50.7615 & 0.0175 & 0.00031 & 0.000170 & 0.000181 \\
14 & 51.01 & 49.21 & 49.24 & 50.81 & 49.37 & 0.9035 & 49.9282 & 0.0181 & 0.00033 & 0.000178 & 0.000191 \\
15 & 49.33 & 48.82 & 49.02 & 50.28 & 49.67 & 0.5734 & 49.4236 & 0.0116 & 0.00013 & 0.000176 & 0.000187 \\
16 & 51.16 & 50.11 & 48.63 & 50.64 & 50.32 & 0.9481 & 50.1721 & 0.0189 & 0.00036 & 0.000185 & 0.000198 \\
17 & 51.04 & 49.48 & 50.35 & 49.19 & 50.22 & 0.7336 & 50.0560 & 0.0147 & 0.00022 & 0.000186 & 0.000199 \\
18 & 49.22 & 50.91 & 50.43 & 51.80 & 51.35 & 0.9919 & 50.7413 & 0.0195 & 0.00038 & 0.000196 & 0.000211 \\
19 & 49.99 & 49.58 & 50.38 & 50.07 & 49.44 & 0.3815 & 49.8924 & 0.0076 & 0.00006 & 0.000189 & 0.000201 \\
20 & 49.26 & 49.01 & 50.17 & 49.55 & 51.07 & 0.8249 & 49.8131 & 0.0166 & 0.00028 & 0.000194 & 0.000206 \\
\hline
\end{tabular}

Table 12: Illustrative example of Phase II dataset. 
$\lambda=0.2 ; B=1 ; m=1 ; \Omega_{D}=[0.5 ; 1)$

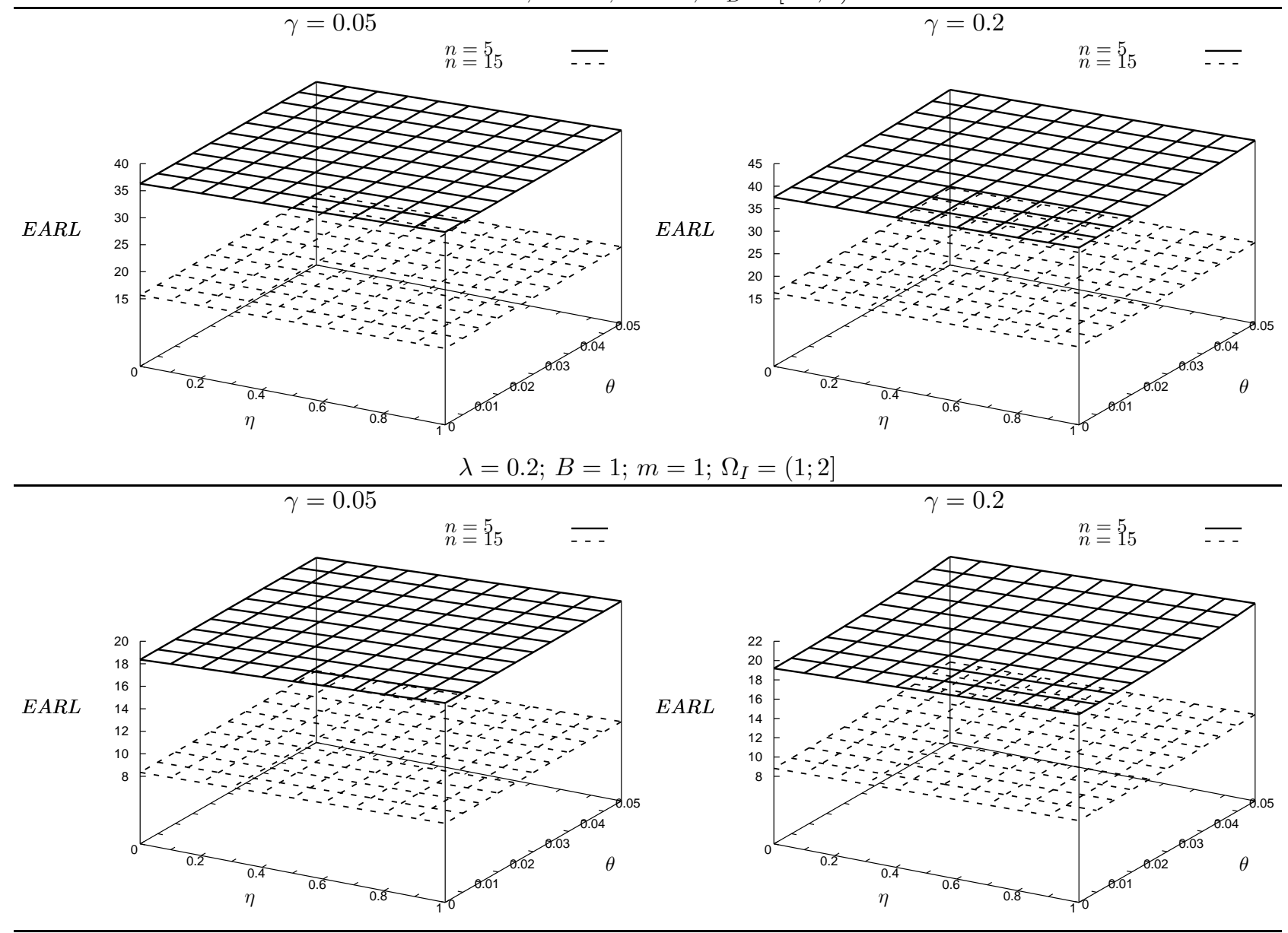

Figure 1: The effect of $\eta$ and $\theta$ on the overall performance of the EWMA- $\gamma^{2}$ control charts in the presence of measurement errors for $\lambda=0.2, A R L_{0}=370.4, B=1, m=1$, $n \in\{5,15\}, \gamma_{0} \in\{0.05,0.2\}$ 


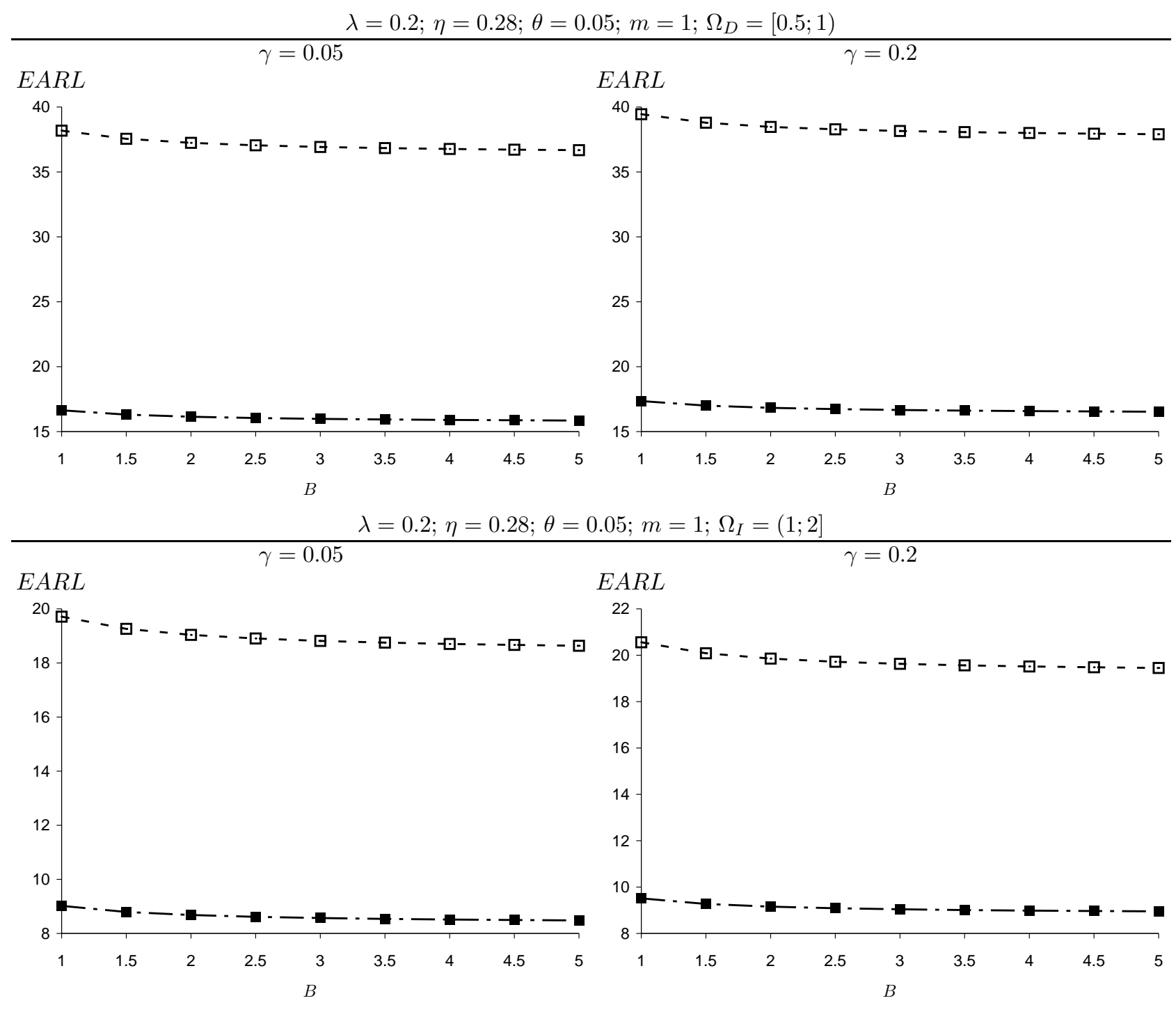

Figure 2: The effect of $B$ on the overall performance of the EWMA- $\gamma^{2}$ control charts in the presence of measurement errors for $n=5$ (-口) and $n=15(-\mathbf{m -}), \lambda=0.2, m=1$, $A R L_{0}=370.4, \eta=0.28, \theta=0.05, n \in\{1,15\}, \gamma_{0} \in\{0.05,0.2\}$ 


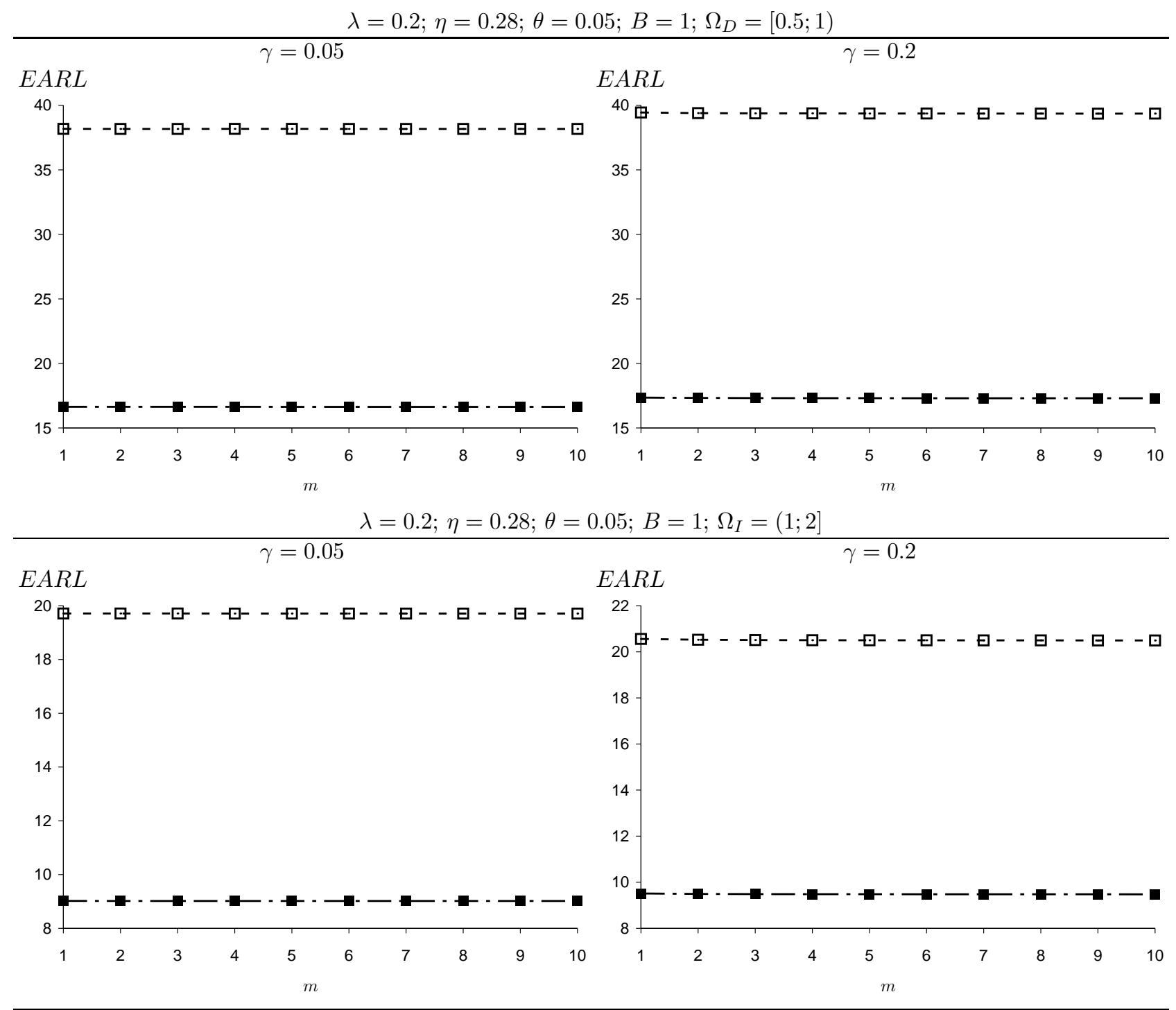

Figure 3: The effect of $m$ on the overall performance of the EWMA- $\gamma^{2}$ control charts in the presence of measurement errors for $n=5(-\square-)$ and $n=15(-\mathbf{m}), \lambda=0.2, B=1$, $A R L_{0}=370.4, \eta=0.28, \theta=0.05, n \in\{1,15\}, \gamma_{0} \in\{0.05,0.2\}$ 


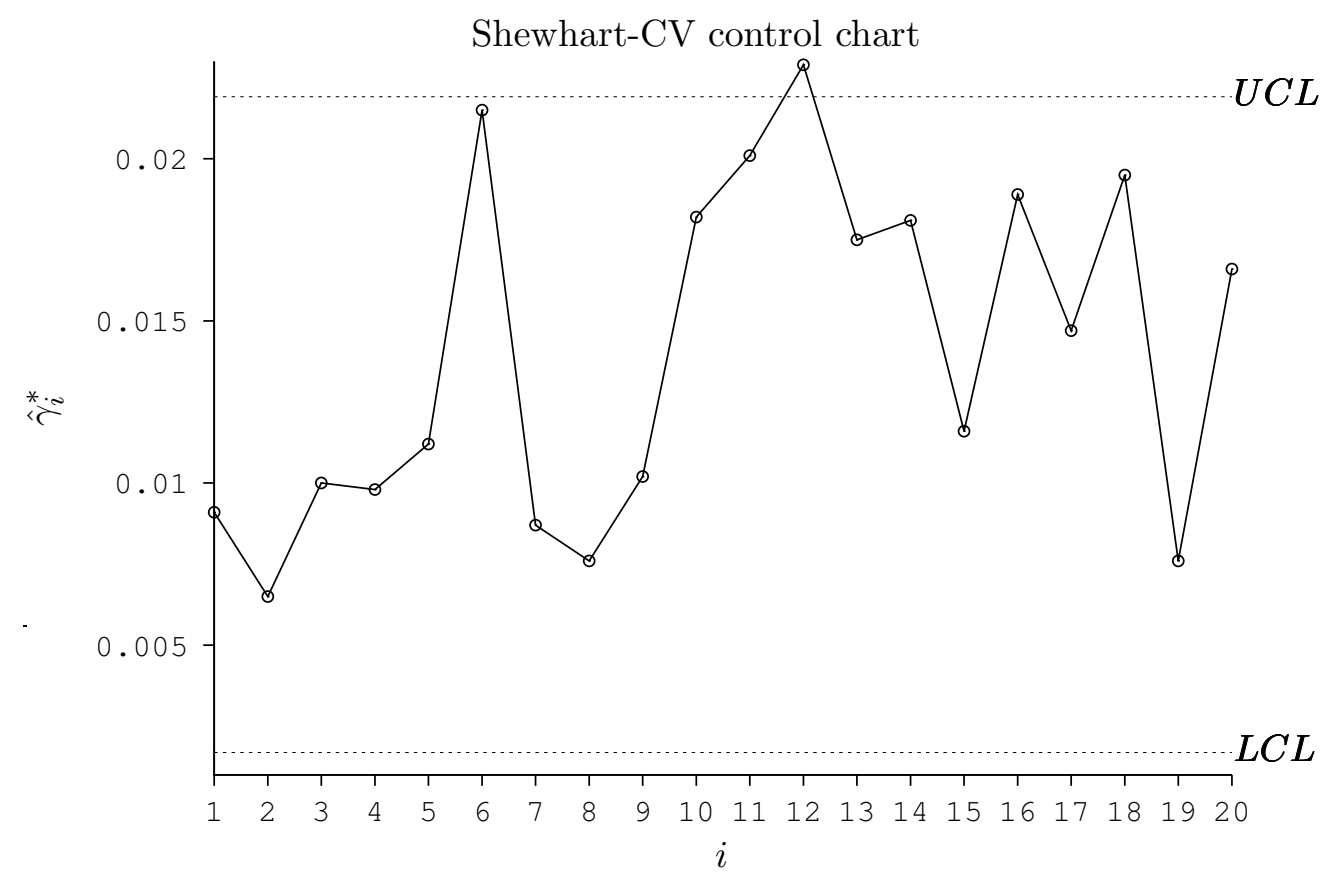

Figure 4: Shewhart-CV control chart applied to the sintering process (Phase II).

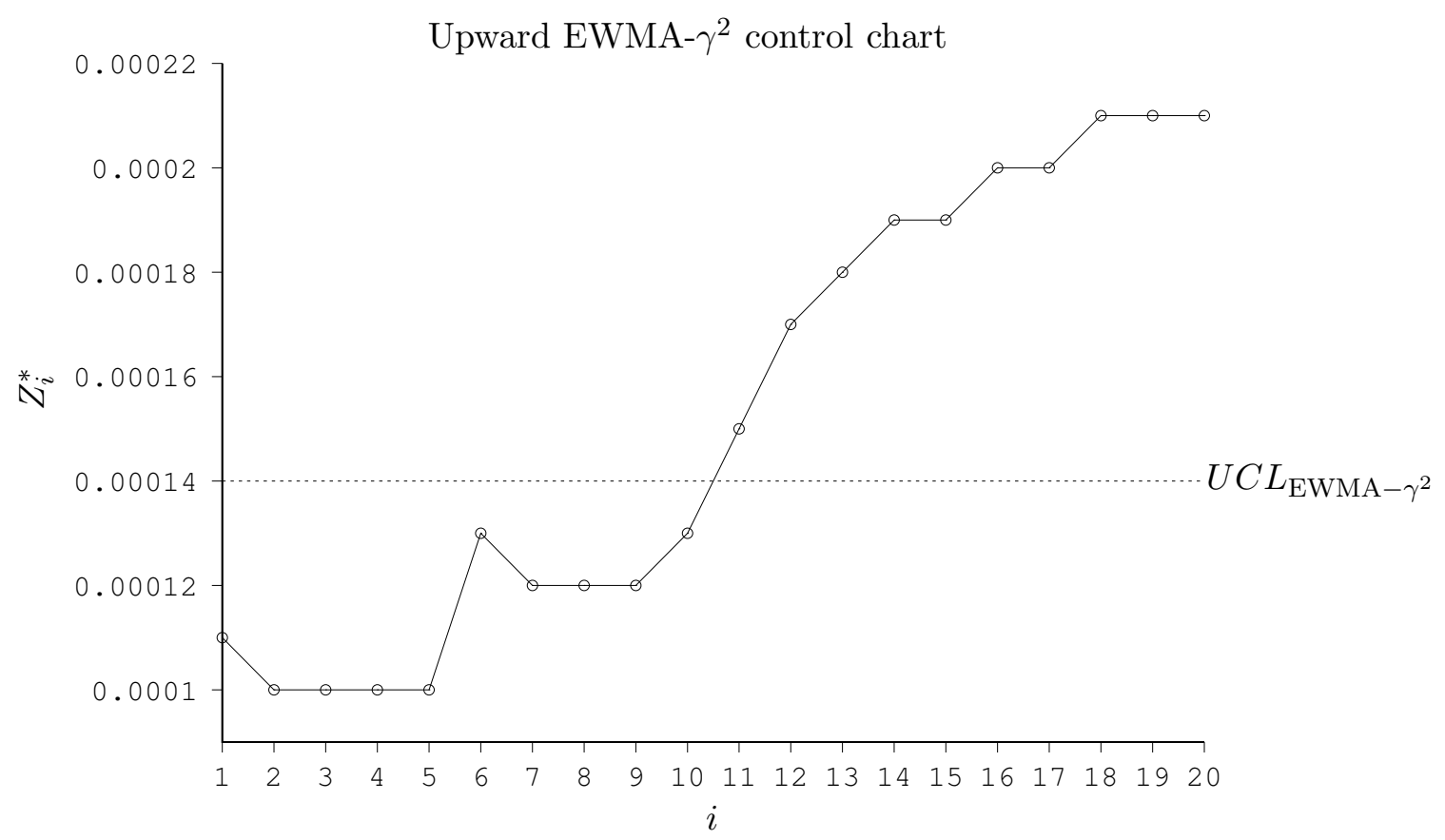

Figure 5: Upward EWMA- $\gamma^{2}$ control chart applied to the sintering process (Phase II). 


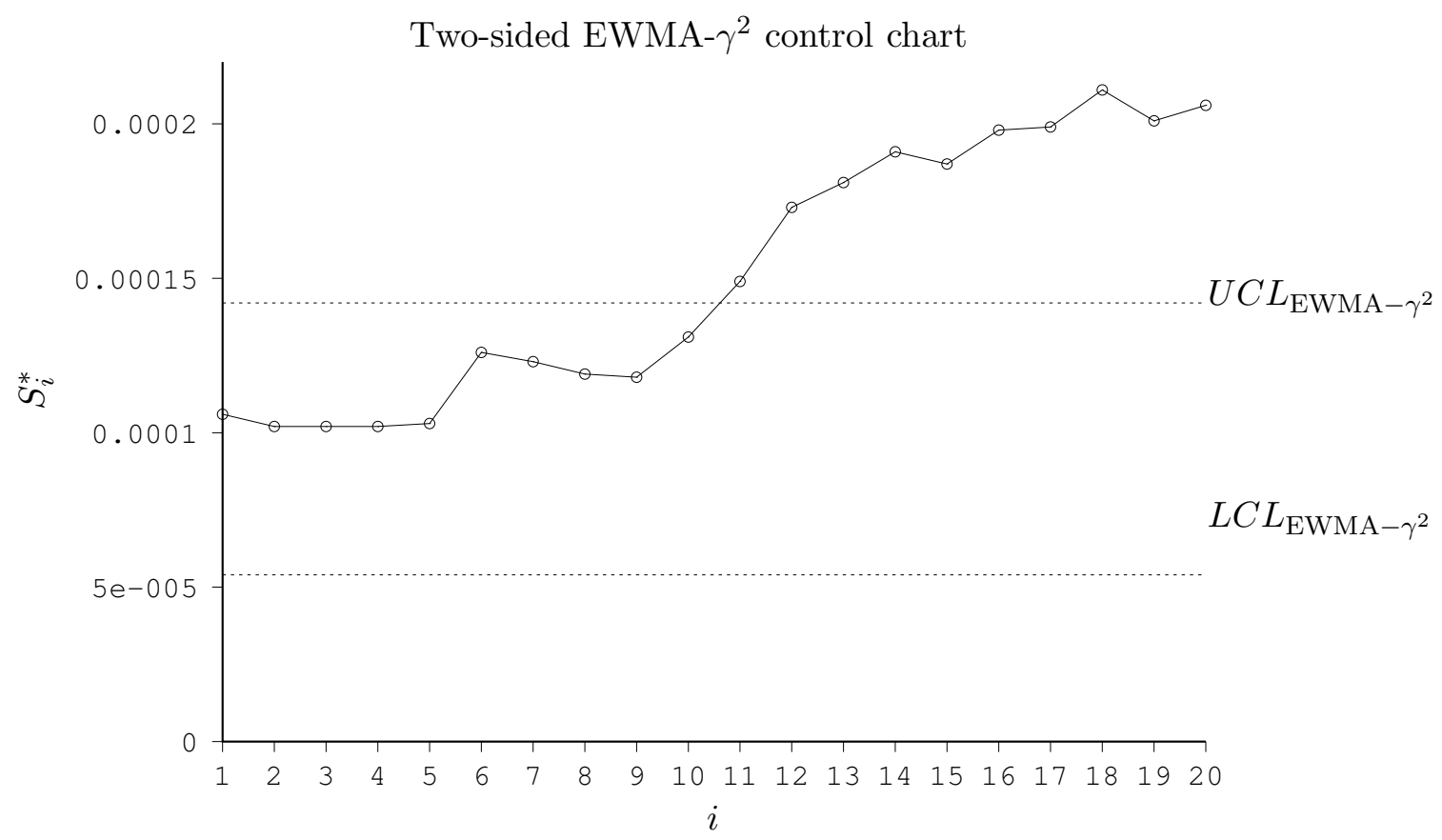

Figure 6: Two-sided EWMA- $\gamma^{2}$ control chart applied to the sintering process (Phase II). 